\title{
Self-Powered Sensors: New Opportunities and Challenges from Two-Dimensional Nanomaterials
}

\author{
Eunkwang Lee ${ }^{1(D)}$ and Hocheon Yoo ${ }^{2, *(D)}$ \\ 1 MLCC Development Team, Samsung Electro-Mechanics, Maeyeong-ro 150, Yeongtong-gu, \\ Suwon 16229, Korea; unisteklee@gmail.com \\ 2 Department of Electronic Engineering, Gachon University, Seongnam 13120, Korea \\ * Correspondence: hyoo@gachon.ac.kr
}

Citation: Lee, E.; Yoo, $\mathrm{H}$.

Self-Powered Sensors: New

Opportunities and Challenges from

Two-Dimensional Nanomaterials.

Molecules 2021, 26, 5056. https:/ /

doi.org/10.3390/molecules26165056

Academic Editor: Minghao Yu

Received: 31 July 2021

Accepted: 17 August 2021

Published: 20 August 2021

Publisher's Note: MDPI stays neutral with regard to jurisdictional claims in published maps and institutional affiliations.

Copyright: (c) 2021 by the authors. Licensee MDPI, Basel, Switzerland. This article is an open access article distributed under the terms and conditions of the Creative Commons Attribution (CC BY) license (https:// creativecommons.org/licenses/by/ $4.0 /)$.

\begin{abstract}
Nanomaterials have gained considerable attention over the last decade, finding applications in emerging fields such as wearable sensors, biomedical care, and implantable electronics. However, these applications require miniaturization operating with extremely low power levels to conveniently sense various signals anytime, anywhere, and show the information in various ways. From this perspective, a crucial field is technologies that can harvest energy from the environment as sustainable, self-sufficient, self-powered sensors. Here we revisit recent advances in various self-powered sensors: optical, chemical, biological, medical, and gas. A timely overview is provided of unconventional nanomaterial sensors operated by self-sufficient energy, focusing on the energy source classification and comparisons of studies including self-powered photovoltaic, piezoelectric, triboelectric, and thermoelectric technology. Integration of these self-operating systems and new applications for neuromorphic sensors are also reviewed. Furthermore, this review discusses opportunities and challenges from self-powered nanomaterial sensors with respect to their energy harvesting principles and sensing applications.
\end{abstract}

Keywords: two-dimensional nanomaterials; optical sensors; self-power; nanostructure devices; sensor devices

\section{Introduction}

Sensors are key ingredients of next-generation electronics in the Internet of Things (IoT) because they contribute to collecting essential signals. The development of various sensors, including photosensors [1-4], gas sensors [5,6], temperature sensors [7-9], and biosensors [10-13] is extensive, which accelerates innovation in new technologies such as the aforementioned IoT. To realize the desired sensing functions, detection performance, such as high sensitivity, robust immunity to noise, and fast response times, should be comprehensively improved [14-17]. As another important performance metric, low power consumption is becoming more and more prominent [18-22]. Modern sensors require miniaturization operating with extremely low power levels to conveniently sense various signals anytime, anywhere, and to show the information in various ways. Thus, technologies combined with desirable materials that can harvest energy from the environment as sustainable, self-sufficient, self-powered sensors are to be considered a promising but necessary direction [23-27].

Meanwhile, the emergence of two-dimensional (2D) nanomaterials has ignited considerable interest in their potential, owing to their atomically thin nature [28] and superior electrical and optical characteristics. These 2D nanomaterials, including graphene [29,30], hexagonal boron nitride ( $\mathrm{hBN}$ ) [31,32], and metal dichalcogenides $\left(\mathrm{MX}_{2}\right)$ [33,34], have a layered structure based on strong in-plane bonds and weak out-of-plane van der Waals (vdW) force. A lot of effort with 2D materials, including hBN insulators, molybdenum disulfide $\left(\mathrm{MoS}_{2}\right)$ semiconductors [35,36], molybdenum diselenide $\left(\mathrm{MoSe}_{2}\right)[35,36]$, tungsten disulfide $\left(\mathrm{WS}_{2}\right)$ [37], tungsten diselenide $\left(\mathrm{WSe}_{2}\right)$ [38], molybdenum ditelluride $\left(\mathrm{MoTe}_{2}\right)[39,40]$, 
and black phosphorus (BP) [41,42], has been put into electrical, optical, and mechanical applications. High transparency resulting from the atomically thin nature and excellent charge transport ability from 2D crystallinity is expediting optoelectronic applications. Furthermore, the mechanical flexibility of these materials offers high compatibility, enabling wearable sensor systems. However, the extremely high voltage operation is considered a strong drawback, limiting sensor system miniaturization and wearable applications in conventional 2D nanomaterials-based sensors. In this light, the operation of self-powered sensors has rapidly emerged and has received a lot of attention in recent years [43-47]. Rather than use an external power source, self-powered sensors receive energy from the surrounding environment. Because there is a variety of environmental energy sources (e.g., piezoelectric [48], triboelectric [49], photovoltaic [50,51], and thermoelectric [50]), a combination of appropriate sensors and energy harvesting can implement a ubiquitous system without power consumption issues.

Here, an overview of self-powered sensors based on 2D nanomaterials is summarized. In Section 2.1, we provide an introduction to 2D nanomaterial-based photodetectors, and we revisit recent advances in their application to self-powered photodetectors. In Section 2.2, we discuss the fundamental principles of piezoelectric nanogenerators (PENG) for self-powered electronics, focusing on their potential applications. In Section 2.3, 2D nanomaterial-based triboelectric nanogenerators (TENG) are introduced. In Section 2.4, we discuss the thermoelectric effects of 2D nanomaterials with an emphasis on their application to self-powered thermoelectric devices. In Section 2.5, we revisit newly developed integration systems consisting of self-powered sensors. In Section 2.6, an emerging application of a neuromorphic self-powered system is introduced, presenting its merits from energy consumption aspects. In Section 3, we discuss the challenges and opportunities from future developments in 2D nanomaterial self-powered sensors.

\section{Self-Powered Sensors}

\subsection{Self-Powered Photodetectors}

Charge carriers (electrons and holes) in 2D materials are able to move quickly on a twodimensional, quantum-confined surface within a thickness of several hundred nanometers. The emergence of graphene has boosted exploration into applications of 2D nanomaterials in photodetectors. Graphene is considered a promising optoelectronic material owing to its amenable energy-level tunability from a variety of chemical doping on its surface [51].

However, non-graphene-like 2D nanomaterials such as transition metal dichalcogenide (TMD), hexagonal hBN, and black phosphorus have gained attention from academia due to the diversity of their chemical compositions and structures. Investigating the phasedependent properties is crucial to explore the 2D TMD applications. The phase and overall material properties of TMD are determined by the coordination of the transition metal and the stacking order between metal and chalcogenide atoms. Typically, the phase of TMD can be categorized with the terms $1 \mathrm{~T}, 2 \mathrm{H}$, and $3 \mathrm{R}$. The number in each term denotes the different layers of the chalcogen atoms and transition metal in the $c$ axis. On the other hand, T, H, and R, respectively, indicate tetragonal, hexagonal, and rhombohedral crystal symmetry. The properties of TMD are greatly affected by phase structure. For example, even for the same $\mathrm{MoS}_{2}$, the $2 \mathrm{H}$ phase represents the n-type semiconductor, and the $1 \mathrm{~T}$ phase represents the metal. The electrocatalytic activity of $1 \mathrm{~T}-\mathrm{MoS}_{2}$ is much higher than that of $2 \mathrm{H}-\mathrm{MoS}_{2}$ in hydrogen evolution reactions. Unlike $2 \mathrm{H}-\mathrm{MoS}_{2}$, the bulk crystals of 1T$\mathrm{MoS}_{2}$ exhibit superconductivity at $4 \mathrm{~K}$. During the $2 \mathrm{H}$-to- $1 \mathrm{~T}$ phase transformation of $\mathrm{MoS}_{2}$, the magnetism changes from diamagnetism to paramagnetism [52]. Typically, in chemical vapor deposition (CVD) processes, when a precursor reacts on substrate-rich dangling bonds, a film begins to grow from the dangling bonds. Since the original 2D TMD lacks dangling bonds as original reaction sites, the out-of-plane film growth is actually inhibited, and it grows in a plate-like shape. Atomically thick 2D TMDs have naturally passivated surfaces from the chalcogenide atoms. These 2D materials are chemically inert, making them suitable for incorporation into silicon structures without lattice mismatch issues [53]. 
Single-layer graphene generally does not have good light absorption, based on the zero band gap property. However, 2D TMD having a direct band gap is high in light absorption and can be implemented in high-performance optoelectronic devices, enabling the generation of excitons through photon illumination, suggesting a new kind of material [54]. TMD is a type of semiconductor with a wavelength of 590 1033 nm or an energy band gap range of $2800 \mathrm{~K} \sim 4900 \mathrm{~K}$ and $1.2 \mathrm{eV} 2.1 \mathrm{eV}$ expressed in converted temperature and energy form. For example, $\mathrm{MoS}_{2}(1.8 \mathrm{eV}), \mathrm{WS}_{2}(2.1 \mathrm{eV}), \mathrm{MoTe}_{2}(1.1 \mathrm{eV})$, and $\mathrm{WSe}_{2}$ $(1.7 \mathrm{eV})$ have a desirable band gap for optoelectronic devices [55]. These properties allow TMD-based photodetectors to identify both visible and NIR ranges, allowing them to be used in thrusters and active layer components in solar cells. In addition, to control the fine electrical properties of the TMD, its band gap can be adjusted in order to manufacture various electronic devices and long-distance temperature sensors in the NIR region by changing the number of layers of the TMD [56].

The utilization of 2D TMDs in photodetectors has been widely investigated. Overcoming technological hurdles, the development of self-powered photodetectors based on 2D TMD has been highly demanding, so far, for the following reasons. Self-powered photodetectors can work without external power sources. This advantage is beneficial for the fabrication of portable, wireless, and low-power devices. In addition, self-powered photodetectors can work independently, so a complex additional power system is not required. The combination of $2 \mathrm{D}$ TMD and a self-powered system would be of great advantage in the construction of lightweight, flexible energy-harvesting devices.

To evaluate the performance of a photodetector, we use various figures of merit, such as detectivity, photoresponsivity, dark current, etc. [57]. In particular, in order to achieve high detectivity $\left(D^{*}\right)$ in the photodetector, it is recommended to use a photosensitive material having an essentially low dark current. Almost all key parameters, such as $\mathrm{D}^{*}$, photoresponsivity (R), and photo-switching on/off ratio, are related to dark current. Traditionally, to suppress dark current, the photodetector (source, drain, and gate) based on the transistor structure is fabricated, and a gate bias is applied to control the charge carrier density of the channel. However, compared structurally to a two-terminal device, a transistor-type three-terminal device is more complicated to make and is inefficient because a gate bias must be continuously applied in terms of energy efficiency. Another way to achieve low dark current is to make a heterojunction TMD. Although this method can effectively improve the performance of the photodetector, it is still technically immature to make the heterojunction in the desired way (precisely and with a large area). Therefore, there is a need to develop a method that can encompass the aforementioned methods [58].

Figure 1 exhibits several working mechanisms for the generation of photocurrent in photodetectors: the p-n junction type, the Schottky junction type, and the photoelectrochemical (PEC) type. The p-n junction and Schottky junction have similar mechanisms in which electron-hole pairs (e.g., excitons) are generated at the interface of the p-n type semiconductor for the p-n junction type (Figure 1a), or at the interface of a metal semiconductor for a Schottky junction, are separated by an applied electrical field (Figure 1b). However, in self-powered mode, spontaneously built-in electrical potential encourages separation of the excitons and drives them to the desired electrodes. On the other hand, the PEC type of mechanism encourages the excitons to separate toward anodes and cathodes via the energy barrier between the electrode and the electrolyte (as shown in Figure 1c). By using the PEC type structure, complete electron-circuit through the oxidation and reduction process in the electrolyte can be implemented in the self-powered PEC photodetector without consuming energy. Figure 1a shows an energy diagram where a space charge layer is formed due to the carrier concentration gradient at the junction when a p-type semiconductor and an n-type semiconductor are "junctioned" together and illuminated from the outside. An internal local electric field is formed in the p-n junction region, and when the light illuminates this part, the photogenerated electrons and holes move via the local electric field of the junction to generate a photocurrent $[59,60]$. 
a $\quad$-n junction type

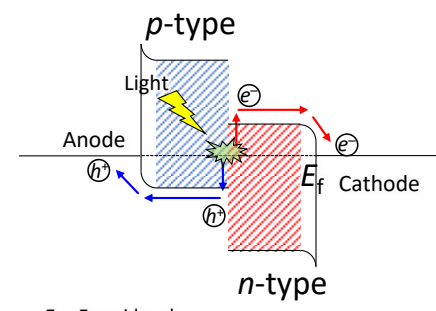

$E_{\mathrm{f}}=$ Fermi level b Schottky junction

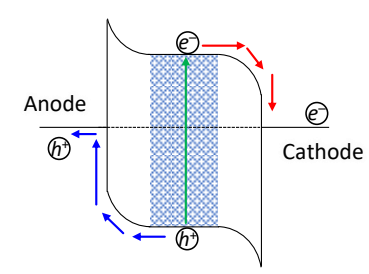

C Photoelectrochemical

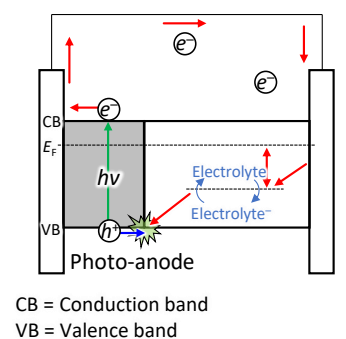

Figure 1. Self-powered photodetector/photovoltaic mechanisms: (a) the p-n junction type, (b) the Schottky junction type, and (c) the photoelectrochemical type.

In particular, various device structures of the p-n junction type in self-powered photodetectors have been reported. The basic structure of a $\mathrm{p}-\mathrm{n}$ junction is a vertical junction in which two different 2D nanomaterials ( $\mathrm{p}$ - and n-semiconductors) make contact. Meanwhile, a lateral junction can be fabricated during the artificial growth of 2D nanomaterials. Typically, heterogeneous reactants are introduced during 2D nanomaterial growth using chemical vapor deposition. Another approach to fabricating photodetectors is the fabrication of a mixed dimensional junction. For example, 1D nanowires or 3D bulk materials can be applied to make a unique junction. Those introduced approaches (vertical, lateral, 2D + 1D, and 2D + 3D junctions) are utilized for specific purposes (performance, flexibility, transparency, ease of fabrication, etc.)

Jin et al. reported the fabrication of self-powered high-performance photodetectors using CdSe nanobelt (NB)/graphene, as shown in Figure 2a [61]. Graphene and Au are utilized as heterogeneous metal electrodes in the photodetector system to form a Schottky junction with CdSe NB. The difference in work function between CdSe $(4.2 \mathrm{eV})$ and graphene $(4.6 \mathrm{eV})$ will result in spontaneous built-in electrical potential. Energy band diagrams in a short circuit and with an external resistor are depicted in Figure $2 b, c$, respectively. When photon energy greater than the band gap energy of CdSe NBs is irradiated, the excited holes and electrons are separated by the built-in field and move to graphene and CdSe NB sides, respectively. The high photoresponsivity $\left(10.2 \mathrm{AW}^{-1}\right)$ and gain (28) of the self-powered CdSe NB/graphene photodetector were obtained. The vertical junction of different $2 \mathrm{D}$ nanomaterials creates an unprecedented different charge transport behavior.

Yang et al. reported a self-driven photodetector using van der Waals nanomaterials $\mathrm{MoS}_{2}$ and GaTe, which are n- and p-type semiconductors, respectively. A GaTe-MoS 2 phototransistor is shown in Figure 2d. A type-II band alignment is made in the GaTe-MoS 2 junction by energy level alignment. In addition, huge built-in potential forces separation of excitons (electron and hole pairs) resulting in self-driven photocurrent. The I-V behavior of vertically stacked GaTe-MoS 2 heterostructures (Figure 2e) shows typical forward bias rectification effects. The I-V behavior obtains an on-off current ratio of about 10. At the positive bias, the built-in potential at the interface between the two materials is greatly reduced, showing a high current. Conversely, at the negative bias, the built-in potential increases, and the off-current becomes smaller. Figure $2 \mathrm{f}$ shows a self-driven operation of the GaTe-MoS 2 heterostructure at the zero bias (source-to-drain) under light illumination. The dynamic behavior of source-drain current is repetitive and maintained for a long period of time [62]. 


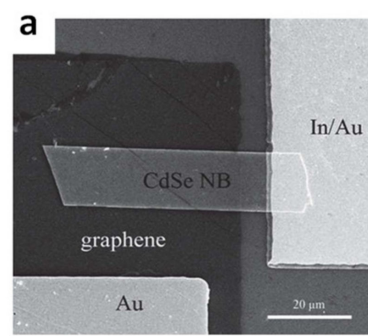

d

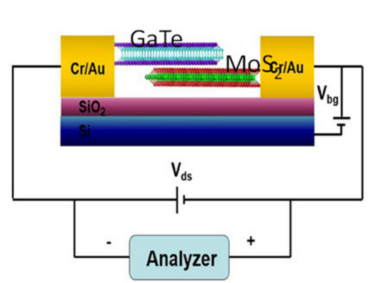

b

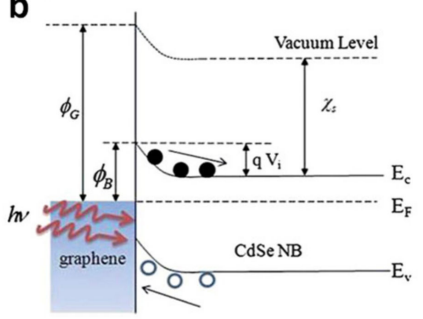

e

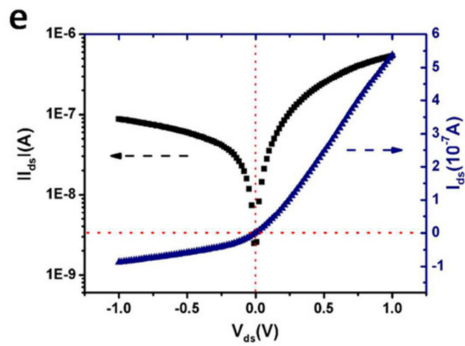

c

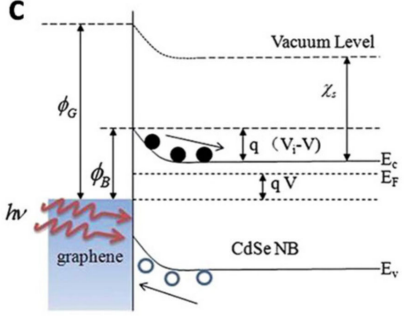

f

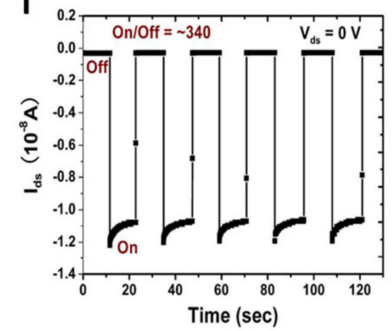

Figure 2. (a) FESEM image of a photodetector; $(\mathbf{b}, \mathbf{c})$ are energy band diagrams for the photodetector under external light irradiation of short circuit and load, respectively. $\Phi_{\mathrm{G}}$ is the work function of graphene, $\Phi_{\mathrm{B}}$ is the Schottky barrier height, $\mathrm{V}_{\mathrm{i}}$ is the built-in potential, $\mathrm{V}$ is the voltage drop of the resistor, $\mathrm{E}_{\mathrm{C}}$ is the conduction band edge, $\mathrm{E}_{\mathrm{V}}$ is the valence band, and $\mathrm{E}_{\mathrm{F}}$ is the Fermi level [61]. Reproduced with permission from [61]. Copyright 2012, Royal Society of Chemistry. (d) A schematic of a GaTe-MoS 2 heterostructure phototransistor. (e) Current-voltage curve of the device (Vds $=-1 \sim 1 \mathrm{~V}$, $\left.\mathrm{V}_{\mathrm{bg}}=0 \mathrm{~V}\right)$. (f) Self-driven photo-switch behavior of the GaTe-MoS 2 heterostructure photodetector $\left(\mathrm{V}_{\mathrm{ds}}=0\right.$ V) [62]. Reproduced with permission from [62], Copyright 2016, American Chemical Society.

Not only the 2D-2D nanomaterial junction, but the construction of 1D-2D and 3D-2D vdW junctions has advantages from several aspects: (i) enhanced light absorption, (ii) a broadened spectral range from UV to IR, (iii) formation of built-in potential, which assists in separating photo-generated excitons, and (iv) more functionality. Wu et al. reported a selfdriven, polarization-sensitive, broadband photovoltaic detector by transferring $2 \mathrm{D} \mathrm{PdSe}$ onto the top of vertically aligned 1D Si nanowire arrays (SiNWA) [63]. Figure 3a is a photograph of an $\mathrm{SiO}_{2}$ substrate, $\mathrm{Pd}$ film on the $\mathrm{SiO}_{2}$, and $\mathrm{PdSe}_{2}$ film grown by direct selenization of the Pd film. The prepared $\mathrm{PdSe}_{2}$ film covered by polymethyl methacrylate (PMMA) was transferred to the SiNWA substrate to form a mixed-dimensional vdW heterostructure. Figure $3 b$ is a cross-sectional SEM image of the $\mathrm{PdSe}_{2} / \mathrm{SiNWA}_{\mathrm{N}}$ heterostructure. A thin film of $\mathrm{PdSe}_{2}$ is placed vertically on top of well-aligned SiNWA. Photocurrent can be detected via on/off light irradiation in various spectral ranges from deep ultraviolet (DUV) to midrange IR (Figure 3c). In particular, the fabricated $\mathrm{PdSe}_{2} / \mathrm{SiNWA}$ photodetector showed a self-powered characteristic that can be detected even when the voltage bias is $0 \mathrm{~V}$. A stable, repeatable, and fast photoresponse from the self-powered photodetector was obtained, showing on/off current ratios (Ion/Ioff) of $10^{4}$ for $265 \mathrm{~nm}\left(2.1 \mathrm{~mW} \mathrm{~cm}^{-2}\right), 10^{6}$ for $980 \mathrm{~nm}$ $\left(56.6 \mathrm{~mW} \mathrm{~cm}^{-2}\right), 10^{2}$ for $2.2 \mathrm{~mm}\left(150 \mathrm{~mW} \mathrm{~cm}^{-2}\right), 3 \mathrm{~mm}\left(35.9 \mathrm{~mW} \mathrm{~cm}^{-2}\right)$, and 26 for $4.6 \mathrm{~mm}$ $\left(125 \mathrm{~mW} \mathrm{~cm}^{-2}\right)$. Thus, the broad photo sensing range of the $\mathrm{PdSe}_{2} / \mathrm{SiNWA}_{\text {hinterostructure }}$ enables high-performance remote sensing, imaging sensors, and night vision. 
a

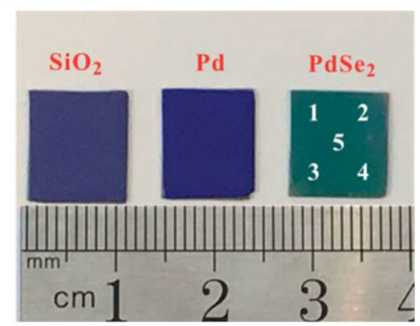

d

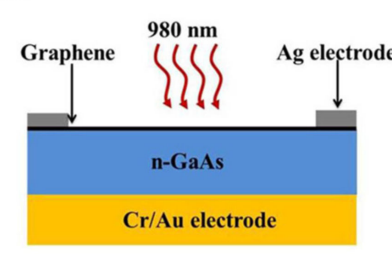

g

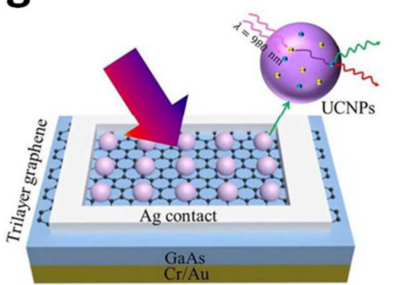

b

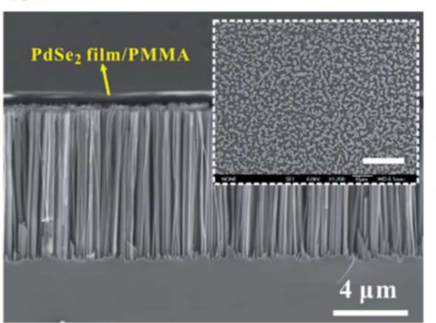

e

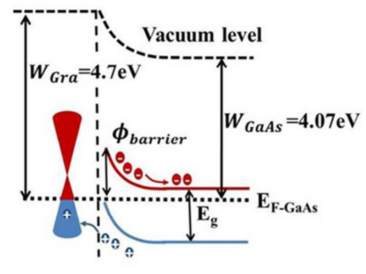

h

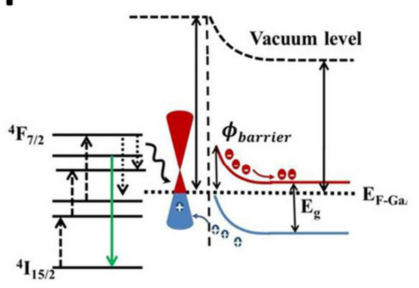

C

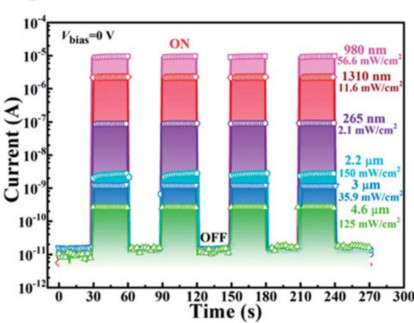

f

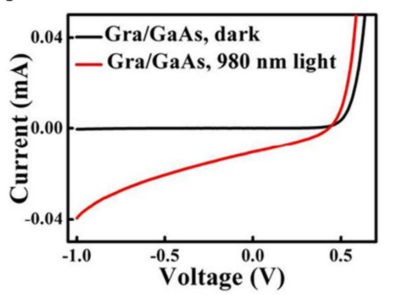

i

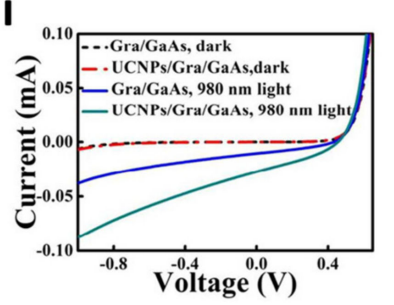

Figure 3. (a) Photographs of the $\mathrm{SiO}_{2} / \mathrm{Si}$ substrate, a Pd film, and a PdSe film formed on the $\mathrm{SiO}_{2} / \mathrm{Si}$ substrate. (b) Cross-sectional SEM image of a $\mathrm{PdSe}_{2}$ heterostructure vertically stacked on SiNWA. The inset shows a top view image of the heterogeneous structure. (c) Time vs. photocurrent plot of $\mathrm{PdSe}_{2} / \mathrm{SiNWA}$ heterostructure devices under illumination at different wavelengths (from $980 \mathrm{~nm}$ to $4.6 \mu \mathrm{m}$ ) at $\mathrm{V}_{\text {bias }}=0 \mathrm{~V}$. Reproduced with permission from [63]. Copyright 2020, Royal Society of Chemistry. (d) A cross-section view of a graphene/GaAs photodetector. (e) Electronic band alignment of the graphene/GaAs heterojunction. (f) I-V behavior of the graphene/GaAs photodetector in the dark (black) and under illumination from a $980 \mathrm{~nm}$ laser (red). (g) Top view of a graphene/GaAs photodetector coated with UCNPs. (h) Electronic band diagram of the UCNP/graphene/GaAs structure. (i) I-V behavior of graphene/GaAs or UCNP/graphene/GaAs photodetectors in the dark and under illumination from a $980 \mathrm{~nm}$ laser. The light illumination power is $55 \mathrm{~mW}$. Reproduced with permission from [64]. Copyright 2018, Royal Society of Chemistry.

$\mathrm{Wu}$ et al. fabricated a graphene/GaAs near-infrared photodetector (NIRPD) decorated with upconverting nanoparticles (UCNPs, $\mathrm{NaYF}_{4}: \mathrm{Yb}^{3+} / \mathrm{E}^{\mathrm{r} 3+}$ ) that can sense a light wavelength of $980 \mathrm{~nm}$ [64]. Figure $3 \mathrm{~d}$ is a schematic of the graphene/GaAs NIRPD consisting of multilayered graphene, n-type GaAs, and $\mathrm{Au}$ and Ag electrodes. Figure $3 e$ is an electronic band diagram of the graphene/GaAs. The work function $\left(\mathrm{W}_{\mathrm{GaAs}}\right)$ of heavily $\mathrm{n}$-doped GaAs $\left(1 \times 10^{18} \mathrm{~cm}^{-3}\right)$ is close to its electron affinity $(\chi=4.07 \mathrm{eV})$. Graphene that shows a p-doped characteristic under an ambient condition has a work function of $4.7 \mathrm{eV}$. Therefore, when they come into contact, electrons diffuse from GaAs to the graphene to reach equilibrium in the electronic concentration gradient, which causes the GaAs energy band to bend upward at the heterojunction interface, with built-in potential created by this. As a result, at the interface of graphene and GaAs, the photo-excited carrier splits into an embedded electrical field under light illumination, generating an increasing reverse current, as shown in the I-V characteristic curve (the red line, Figure 3f). On the other hand, the graphene/GaAs NIRPD shows good rectification behavior in the dark (the black line). Figure $3 g$ is a schematic of the UCNPs/graphene/GaAs NIRPD structure. UCNPs dispersed in an organic solvent are coated on the graphene surface. 
Figure $3 \mathrm{~h}$ is an electronic band diagram of a graphene/GaAs NIRPD with UCNPs on the graphene surface. As shown in the energy band diagram, there are three mechanisms involved in an enhanced photosensing characteristic. The first is radiative energy transfer (RET). Due to the presence of UCNP, upconversion luminescence from the UCNP results in multiple photon pumping with low energy. The second mechanism related to the enhanced photosensing behavior is non-radiative RET. Absorbed light directly creates electron excitation in graphene. Lastly, an antireflection effect caused by UCNPs reduces the reflectance, which accompanies an efficient collection of light. I- $V$ curves of graphene/GaAs and UCNPs/graphene/GaAs photodetectors in the dark and under illumination from a $980 \mathrm{~nm}$ laser are compared in Figure 3i. In dark conditions, both graphene/GaAs and UC$\mathrm{NPs}$ /graphene/GaAs photodetectors exhibit electrical current as low as $2.75 \times 10^{-10} \mathrm{~A}$ in a grounded state. Contrarily, clearly increased reverse bias photocurrent for graphene/GaAs and UCNPs/graphene/GaAs photodetectors was obtained under illumination at $980 \mathrm{~nm}$. Compared to a graphene/GaAs photodetector, UCNPs/graphene/GaAs photodetectors show greatly increased short circuit current by a factor of 2.6 times.

Zhou et al. reported an asymmetrical metal-semiconductor-metal (MSM) photodetector by using graphene and $\mathrm{Au}$ as the electrode contacting the $\mathrm{WSe}_{2}$ flake. The graphene and $\mathrm{WSe}_{2}$ were vertically stacked in part to form the van der Waals contact via the dry-transfer method [65]. Figure $4 \mathrm{a}$ is an illustration of a graphene-WSe 2 -Au photodetector on a $\mathrm{SiO}_{2} / \mathrm{Si}$ wafer substrate. As shown in the illustration, graphene and $\mathrm{WSe}_{2}$ are superimposed on each other in the middle. Figure $4 \mathrm{~b}$ shows an energy band diagram of the photodetector with a graphene-WSe 2 -Au metal-semiconductor-metal (MSM) structure in the presence of light. It shows a large Schottky barrier difference between graphene and Au. In particular, in order to avoid surface material defects that can be induced through the process, and unavoidable doping and Fermi level pinning at the interface, a PDMS-assisted dry transfer method was utilized without the use of a metal deposition system. The $\mathrm{WSe}_{2}$ flake was at first transferred onto the pre-patterned Au electrodes, and the multilayer graphene flake was sequentially transferred onto the $\mathrm{WSe}_{2}$ flake. The fabricated asymmetric photodetector composed of graphene-WSe $e_{2}-\mathrm{Au}$ has self-powered characteristics, as evidenced by the $\mathrm{I}_{\mathrm{sc}}-\mathrm{V}_{\mathrm{oc}}$ curve under light illumination, at a variety of wavelength ranges $(405,532$, $780 \mathrm{~nm}$ ) as shown in Figure 4c-e. Figure $4 \mathrm{f}$ summarizes the dependence of $\mathrm{V}_{\mathrm{oc}}$ and the corresponding light power density on the gate voltage bias under a $780 \mathrm{~nm}$ wavelength. In conclusion, the graphene-WSe ${ }_{2}-\mathrm{Au}$ photodetector demonstrated similar gate-tunability through the change of light power intensity. 
a

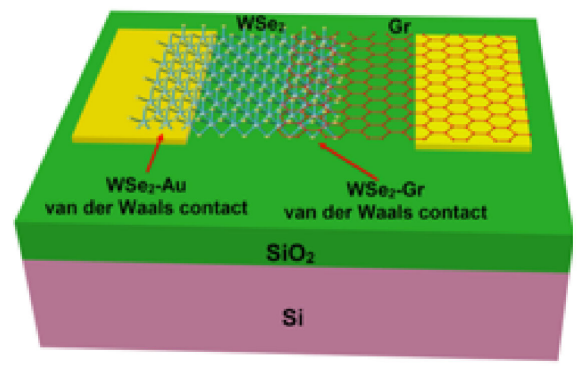

Gr electrode dry transfer

C

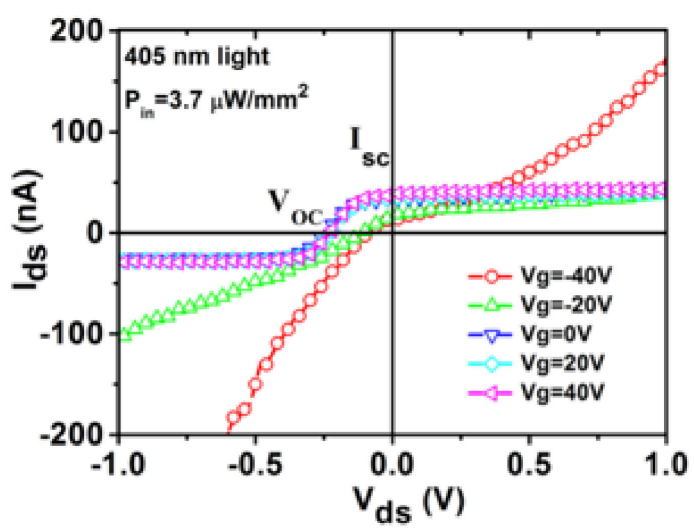

e

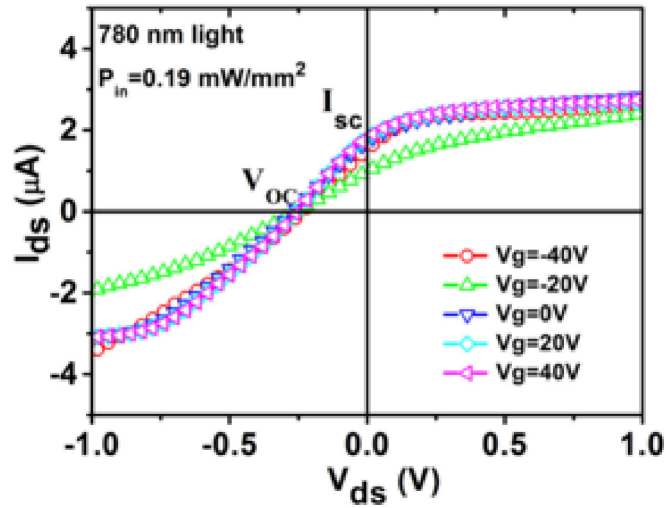

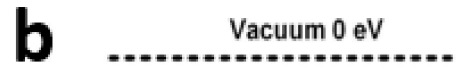

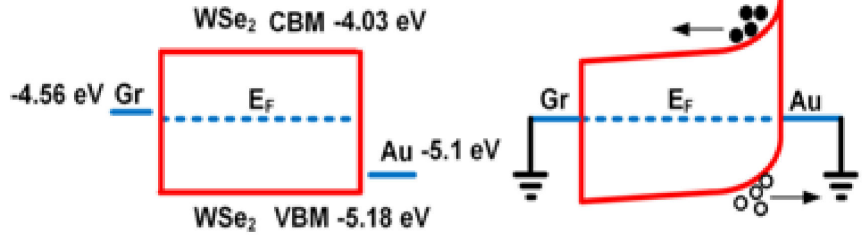

d

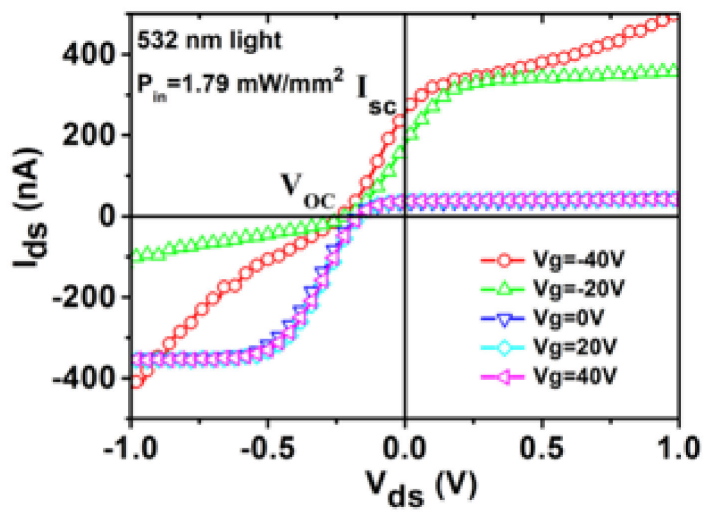

f

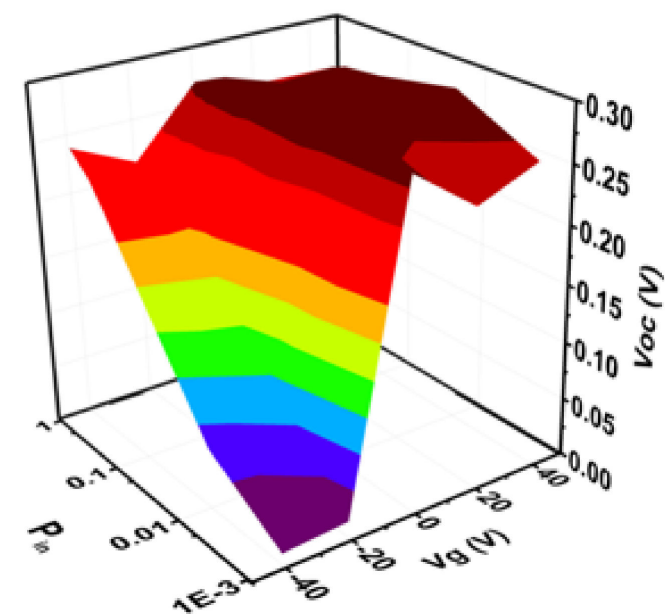

Figure 4. (a) A simple illustration of a graphene-WSe $\mathrm{W}_{2}-\mathrm{Au}$ photodetector. (b) Energy band diagram of a device composed of graphene-WSe 2 -Au. Graphene and Au were used as electrodes. Current-voltage characteristics of graphene-WSe $\mathrm{C}_{2}-\mathrm{Au}$ photodetectors are shown at wavelengths of (c) $405 \mathrm{~nm}$, (d) $532 \mathrm{~nm}$, and (e) $780 \mathrm{~nm}$. (f) Dependence of the open-circuit voltage (Voc) on gate voltage bias and light power density at $780 \mathrm{~nm}$ illumination. Reproduced with permission from [65]. Copyright 2020, Springer Nature.

Kumar et al. demonstrated a graphene/p-type Si-based electronic circuit modulated by a light illumination logic gate [66]. The graphene/p-type Si-based electronic circuit operates over a broad spectral range from ultraviolet to a near-infrared range under a zero bias. Figure 5a shows an electronic energy band diagram of the graphene/p-type Si-based electronic circuit. Due to the Fermi level pinning of graphene and p-type Si, and the formation of a Schottky barrier, energy band bending occurs at the interface between them, which might force the separation of excitons. According to the principle, a photon-induced electro-gating effect on the graphene/p-type Si can be observed on a selective area with a 
small spot size laser source, as shown in Figure $5 b$. Photon-induced potential can break the symmetry between the metal electrodes. Figure $5 \mathrm{c}$ is a schematic of a photon-triggered transistor (PTT). By modifying the appropriate driving source (light intensity, $\mathrm{P}_{\mathrm{L}}$ ) and optoelectronic gate $\left(\mathrm{P}_{\mathrm{R}}\right)$ optical inputs, the PTT offers a way to inject the photogenerated carriers locally. Figure $5 \mathrm{~d}$ shows the current-illuminance intensity $\left(\mathrm{I}-\mathrm{P}_{\mathrm{L}}\right)$ curve as a function of light intensity from 0 to $1 \mathrm{~mW} \mathrm{~cm}{ }^{-2}$. The device exhibits a current of up to $190 \mu \mathrm{A}$ when powered only with light, and with no voltage applied. In particular, to mimic the human retina, we made an electronic device that mimics cells based on the voltage difference, as shown in Figure 5e, and that determines the polarity of the current according to the local location of the light. Thus, incident light near the left junction $\left(P_{L}>0\right.$ and $\left.P_{R}=0\right)$ generates a current in both directions, as shown in Figure 5f. Conversely, when $P_{L}=0$ and $P_{R}>0$, the current changes in the negative direction.

a

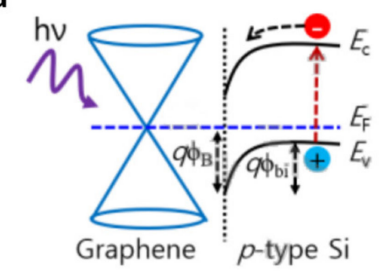

b
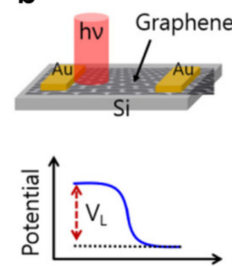
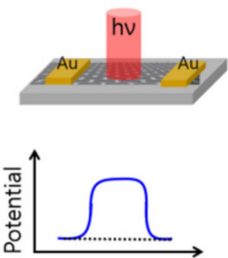
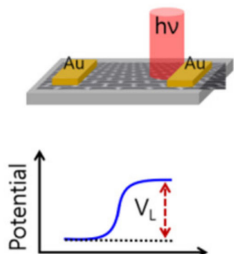

C

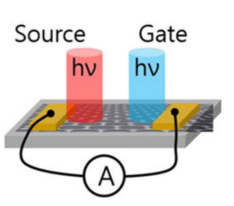

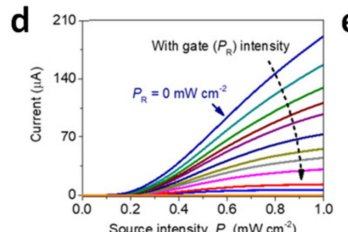

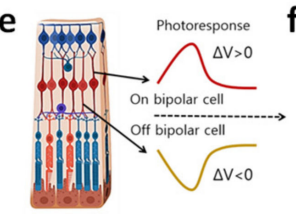

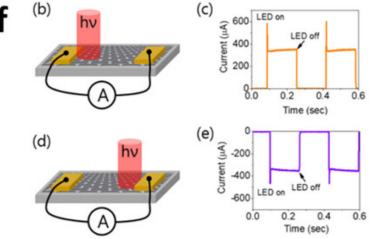

Figure 5. (a) Simplified energy band diagram of a graphene/p-type Si heterostructure. (b) The operational principle of the fabricated graphene/p-type Si device, and the location of light irradiation. (c) Illustration of a photon-triggered transistor (PTT) using two light sources. (d) The current-source light characteristic as a function of gate light intensity. (e) Functional schematic of the in vivo retinal information processing system. (f) A two-terminal device, illuminating the left- and right-electrode sides. The illumination conditions were $\lambda=420 \mathrm{~nm}, 2 \mathrm{~mW} \mathrm{~cm}{ }^{-2}$. Reproduced with permission from [66], Copyright 2020, Elsevier.

\subsection{Self-Powered Piezoelectric Devices}

The acquisition of energy in our daily lives to run electronic gadgets is an intriguing issue when it comes to satisfying the energy needs of the Internet of Things (IoT). Nanogenerators are a promising platform for harvesting many forms of energy surrounding us. Nanogenerators can be related to vibrational energy, motion of the human body, mechanical triggering of tire rotation, wind flow energy, solar energy, blue energy, thermoelectric energy, and so on. Nanogenerators based on piezoelectric and triboelectric effects (PENG and TENG) are fascinating in this regard because they might combine several diverse nanogenerators into a single unit, which can utilize numerous energy sources simultaneously, enabling the use of any handy ambient energy at any time [67].

The mechanism underlying PENG operations is the direct piezoelectric effect. The piezoelectric effect is the electrical polarization of dielectric materials. In a direct piezoelectric mechanism, a force is applied along the asymmetric direction of the dielectric material, and a charge is generated on the opposite surface. When the applied force is removed, the dielectric material returns to its original state (Figure 6a) [68]. On the other hand, the fundamental operation of a TENG is a sequential state between contact and separation to realize the constant transfer of a charge, as shown in Figure 6b [67]. The charge-generating and -trapping materials make contact with each other, and dipoles are generated. Then, when the deformation is released and the materials detach, the opposite charges of the two 
surfaces will separate, so that these opposite triboelectric charges will generate an electric field between them, and thus, a difference of potential is induced between the top and bottom electrodes. Finally, the electrons will be driven to flow from one electrode to the other through the external load.

\section{a Piezoelectric nanogenerator}

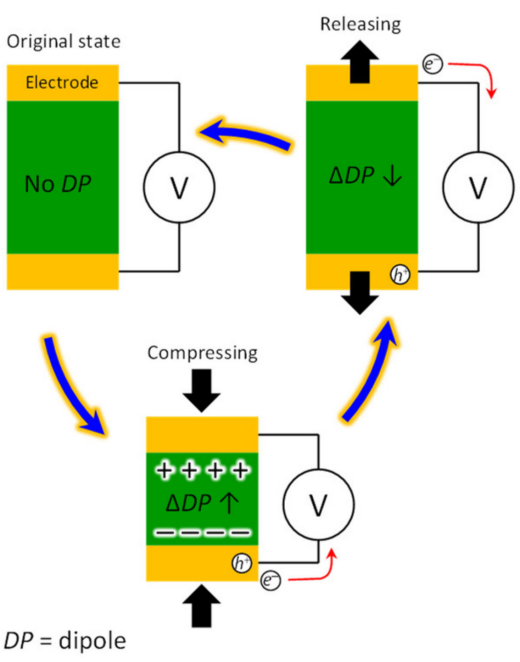

b Triboelectric nanogenerator

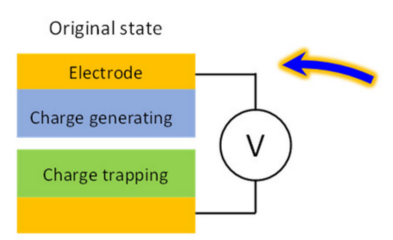

Releasing $\rightarrow$ Detached
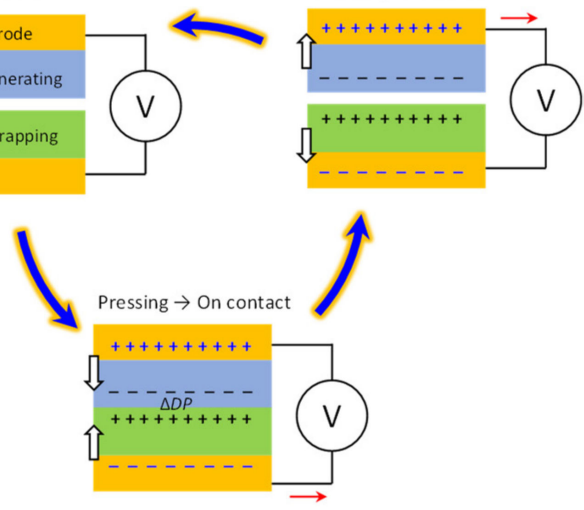

Figure 6. PENG- and TENG-based sensor mechanisms.

Interestingly, 2D monolayer nanomaterials have great potential in high-performance PENG and TENG. Unlike bulk materials, which are centro-symmetric structures, monolayer $\mathrm{MoS}_{2}$ is strongly piezoelectric under the strain-induced lattice distortion and charge polarization. Not only $\mathrm{MoS}_{2}$, but also a broad range of 2D materials, including TMDs, graphene, and monolayer group-IV and -III monochalcogenides, exhibit excellent piezoelectric effects depending on the PENG and TENG structure [69]. Regarding the fabrication of triboelectric devices, TENG performance can be expected to improve mainly from two aspects when 2D nanomaterials are used. When 2D nanomaterials are used, an improved induced charge generation rate can be expected, and the low internal resistance can contribute to an excellent output. In addition, the high specific area of 2D nanomaterials allows the formation of many charge-trapping sites [49].

The piezoelectric power output from a single-layer $\mathrm{MoS}_{2}$ PENG is highly dependent on the atomic orientation. Kim et al. prepared $\mathrm{MoS}_{2}$ via the CVD method and confirmed that the piezoelectric modulus was different along the armchair (Mo and S parallel) and zigzag (Mo and S on the same line) directions (Figure 7a,b) [70]. Figure 7c shows a high distinct piezoelectric response (piezoelectric coefficient, $3.78 \mathrm{pm} / \mathrm{V}$ ) for monolayer $\mathrm{MoS}_{2}$ and $\alpha$-quartz, as a function of the voltage magnitude in the armchair direction, compared to $\alpha$-quartz. In contrast, the piezoelectric response in the zigzag direction showed a lower response, compared to $\alpha$-quartz, with a piezoelectric coefficient of $1.38 \mathrm{pm} / \mathrm{V}$ (Figure 7d). 


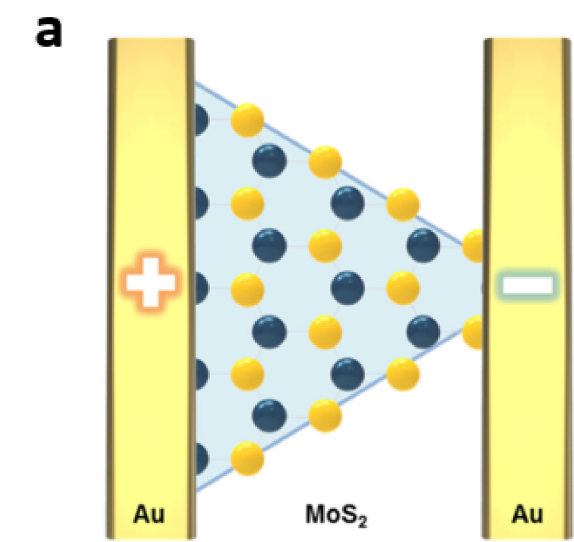

C

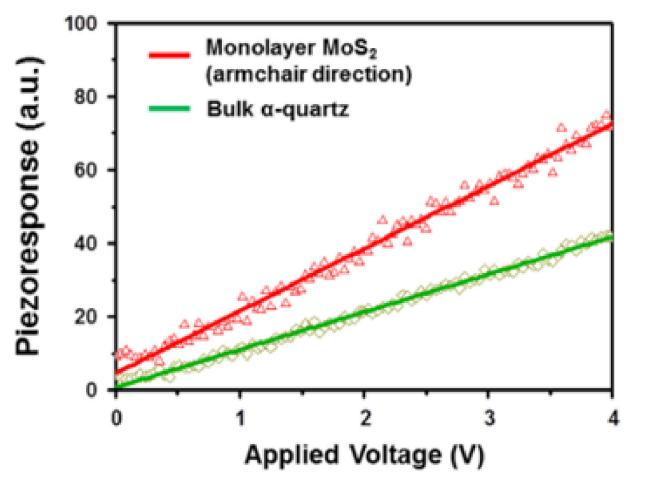

$\mathbf{e}$

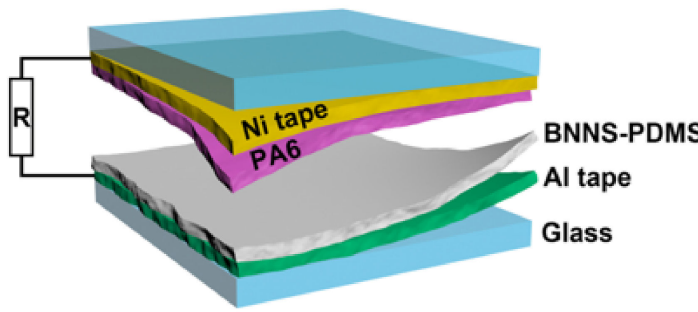

b

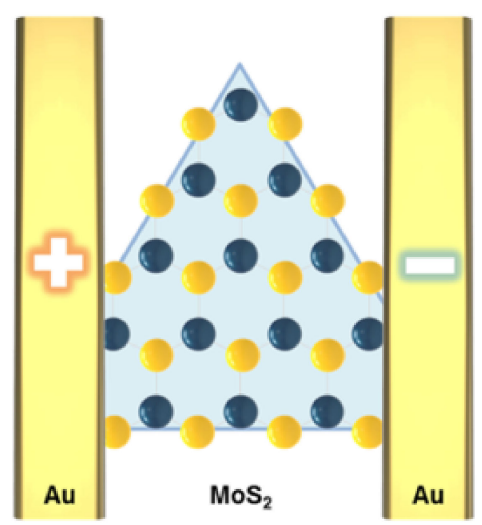

d

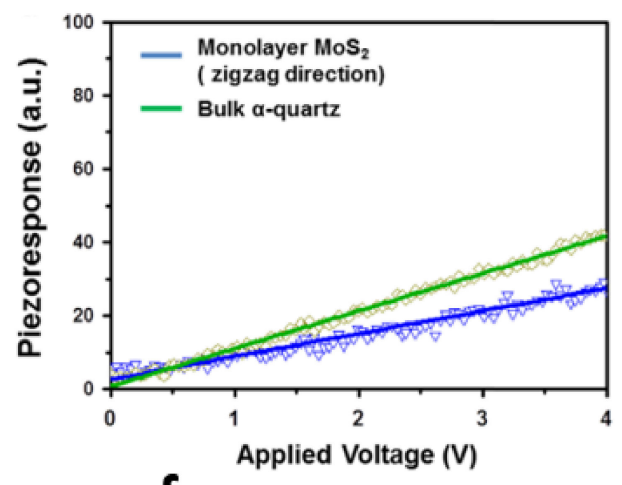

$\mathbf{f}$

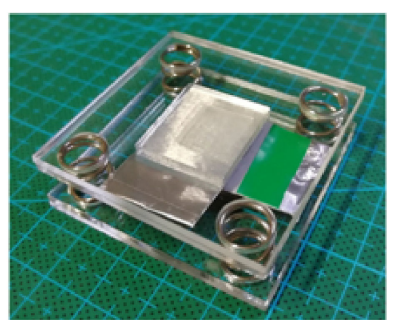

Figure 7. Corresponding piezoelectric modulus (d11) for monolayer $\mathrm{MoS}_{2}$ flakes and electrode positions in (a) the armchair direction and (b) the zigzag direction. High distinct piezoelectric response for monolayer $\mathrm{MoS}_{2}$ and $\alpha$-quartz in (c) the armchair direction and (d) the zigzag direction. Reproduced with permission from [70], Copyright 2016, Elsevier. (e) A 3D schematic and (f) a photograph of the integrated PTEG device. Reproduced with permission from [71], Copyright 2020, Elsevier.

The presence of piezoelectricity in multilayer $\mathrm{hBN}$ has been predicted theoretically, and the piezoelectric voltage coefficient of $2.35 \times 10^{-3} \mathrm{VmN}^{-1}$ has been experimentally confirmed. Applying this, Kuang et al. demonstrated the piezoelectric effect of the hBN nanosheet (BNN)-PDMS composite membranes [71]. The schematic device structure and photos of the BNNS-PDMS/PA6 PTEG are shown in Figure 7e,f. Thus, a piezoelectric and triboelectric nanogenerator (PTEG) was fabricated by grafting a PA6 membrane onto a BNNS-PDMS membrane.

Dai et al. investigated the morphological structure of grain boundaries (GBs) in $\mathrm{MoS}_{2}$ flakes and observed the improved piezoelectric effect through the existence of the GBs [72]. The measured piezoelectric output power of the $\mathrm{MoS}_{2}$ with GB (GB-MoS 2 ) exhibited $50 \%$ higher than a single-crystal $\mathrm{MoS}_{2}\left(\mathrm{SC}-\mathrm{MoS}_{2}\right)$. The piezoelectric response was recorded as shown in Figure 8a-d. The output current density of SC-MoS 2 with a zigzag orientation is displayed in Figure $8 \mathrm{~b}$, showing that the current density induced by deformation of the device is about $0.05 \mathrm{pA} / \mu \mathrm{m}^{2}$. On the other hand, the output current density of SC-MoS ${ }_{2}$ with an armchair orientation is displayed in Figure 8c, showing that 
the current density induced by deformation of the device is about $0.08 \mathrm{pA} / \mu \mathrm{m}^{2}$, which indicates that the armchair orientation of $\mathrm{MoS}_{2}$ results in a slightly higher value for current density. As for the GB-MoS${ }_{2}$-based device shown in Figure 8e, the current densities are about $0.07-0.12 \mathrm{pA} / \mu \mathrm{m}^{2}-$ much larger than from the SC-MoS 2 . The statistical results of current density were obtained from those devices, as shown in Figure $8 \mathrm{f}$. In conclusion, when more GB is present in $\mathrm{MoS}_{2}$, it shows a higher current output result, indicating that the contribution of piezoelectric enhancement by GB is significant.
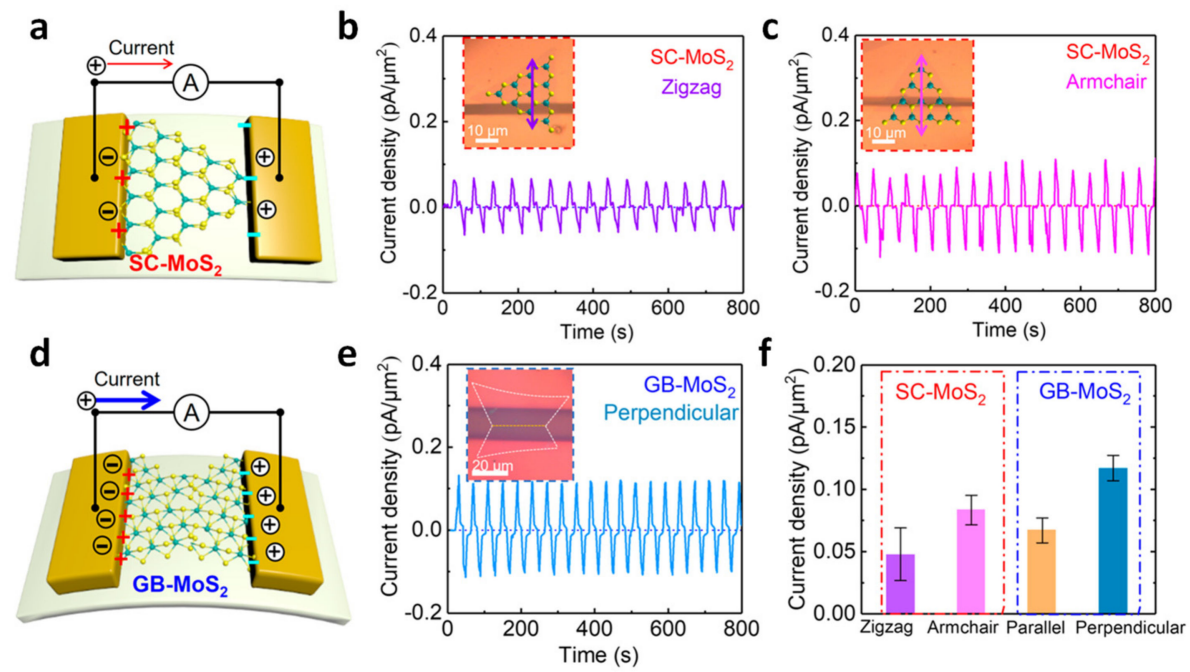

Figure 8. (a) Illustration of the SC-MoS flake-based flexible piezoelectric device. The real-time current output of SC-MoS 2 piezoelectric devices along (b) zigzag and (c) armchair orientations. (d) Illustration of a GB-MoS 2 flake-based flexible piezoelectric device. (e) Current output obtained from the GB-MoS 2 piezoelectric device perpendicular to the grain boundary direction. (f) Current densities of $\mathrm{MoS}_{2}$-based piezoelectric devices under various conditions under the same experimental conditions. Reproduced with permission from [72], Copyright 2020, American Chemical Society.

\subsection{Self-Powered Triboelectric Devices}

The triboelectric effect is a type of electrification induced by contact between different electrostatically charged materials. Recently, TENGs have received lots of attention from academia and industry due to an increased interest in renewable energy that can convert available mechanical energy into electrical energy. Figure $9 \mathrm{a}$ is the simplest form of TENG. It works when contact between two different dielectrics occurs, and electrodes are deposited at both ends to extract electrons generated by friction. Physical contact between these structures creates opposing charged surfaces in a vertical manner. Figure $9 b$ has the same structure as Figure 9a, but when two dielectric films come into contact, they generate frictional charges through a sliding motion in the lateral direction. Lateral sliding begins to flow in an external circuit to balance the field generated by the triboelectric electrons. In this case, AC output is generated via periodic rubbing from side to side. For fabrication of a simpler structure, single-electrode TENG can be fabricated, which consists of a bottom electrode and an active component (Figure 9c). In the single-electrode system, if the distance between the electrodes changes, the electrical potential is disturbed. Then, electrons will be transported in order to balance electrical charges. On the other hand, certain dielectric layers can be established on top of two separated metal electrodes without contacting each other. Moving the dielectric material back and forth, asymmetric charge distributions are generated, which might cause an electrical current flow (Figure 9d). The oscillation of the electrons caused by moving the dielectric produces electrical power. In this case, the freestanding dielectric layer does not need direct contact. A rotational TENG device can be designed without any mechanical contact between the dielectric and metal electrodes Thus, it is a good approach to enhancing the durability of the nanogenerator. 


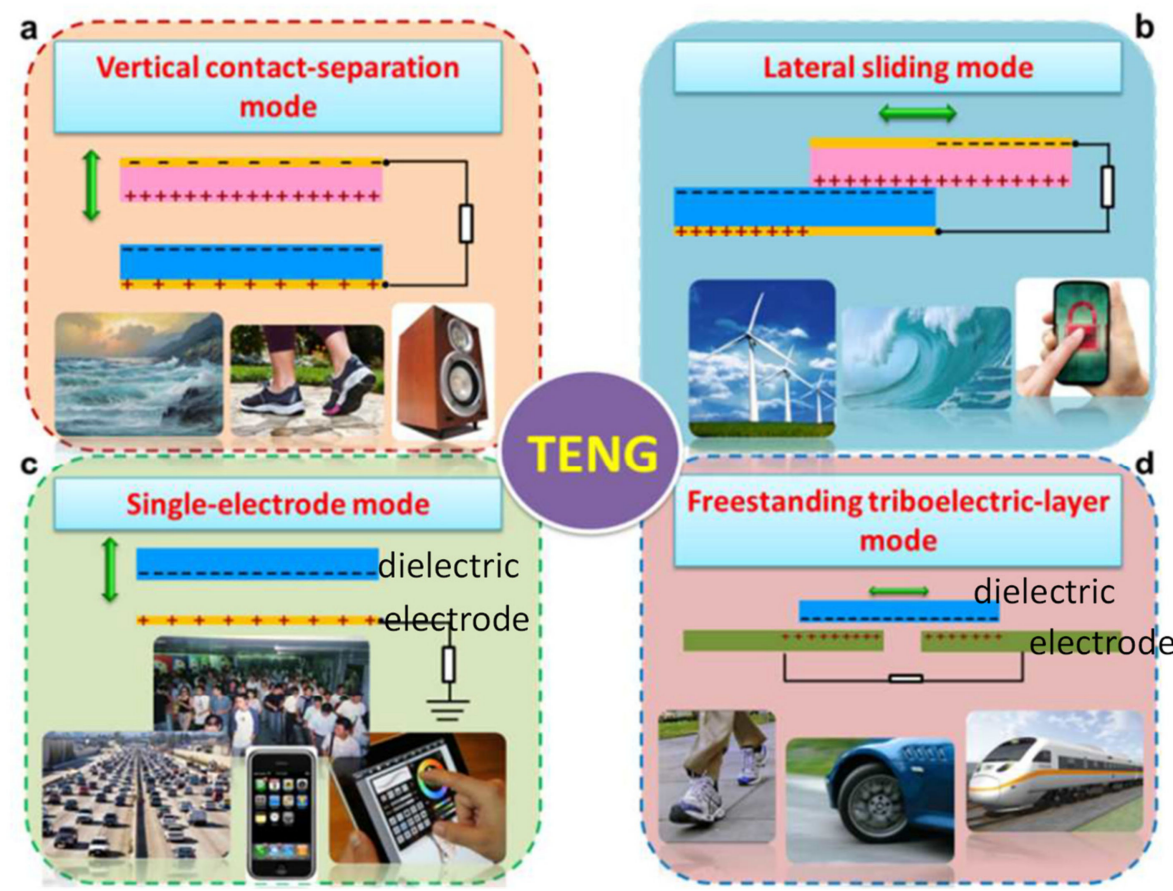

Figure 9. Four basic modes of operation in triboelectric nanogenerators: (a) vertical contact separation mode, (b) side-sliding mode, (c) single-electrode mode, and (d) standalone mode. Reproduced with permission from [73], Copyright 2015, Royal Society of Chemistry.

Park et al. characterized TENG devices consisting of either flat or crumpled $\mathrm{MoS}_{2}$ layers. The crumpled $\mathrm{MoS}_{2}$ was synthesized by laser-directed thermolysis [74]. Figure 10a is a schematic for fabrication of the flat and crumpled $\mathrm{MoS}_{2}$ using a laser line-scribing machine on an $\left(\mathrm{NH}_{4}\right)_{2} \mathrm{MoS}_{4}$-coated $\mathrm{Si}$ wafer substrate. The photothermal interaction induced by the laser creates sufficient heating of the $\left(\mathrm{NH}_{4}\right)_{2} \mathrm{MoS}_{4}$ film, which forms a $2 \mathrm{D}$ $\mathrm{MoS}_{2}$ layer. Interestingly, if the irradiation intensity of the laser is stronger than $2.62 \mathrm{Jcm}^{-2}$, the crumpled structure of the $\mathrm{MoS}_{2}$ layer is formed. As illustrated in Figure 10b, either the flat structure or the crumpled structure of the $2 \mathrm{D} \mathrm{MoS}$ is utilized to fabricate a contactseparation type TENG to figure out the nanostructure effect of 2D MoS 2 layers. PDMS, which is a commercial silicone elastomer, is used as a counterpart triboelectric material. As shown in Figure 10c, in crumpled $\mathrm{MoS}_{2}$, two operating principles are involved: contact charging and electrostatic induction. In the initial state (i), there is an air gap between the $\mathrm{MoS}_{2}$ and the Al/PDMS composite electrode, so that no electron flow occurs, and in (ii), when contact between the two layers occurs, a triboelectric charge is formed on the surface due to electron transfer from PDMS to the $\mathrm{MoS}_{2}$. Compared to the less crumpled and most crumpled $\mathrm{MoS}_{2}$, the most crumpled $\mathrm{MoS}_{2}$ results in a greater shear friction effect on the device. On the other hand, a flat $\mathrm{MoS}_{2}$ produces almost no shear friction. When a negative triboelectric charge is generated on the most crumpled $\mathrm{MoS}_{2}$ surface, the PDMS surface is converted to a positively charged state. Thereafter, as we separate the Al/PDMS electrode from $\mathrm{MoS}_{2}$, in (iii), the potential within the separation generates a current from the $\mathrm{MoS}_{2}$ side to the $\mathrm{Al} / \mathrm{PDMS}$ electrode side throughout the external circuit to compensate for the friction charge generated by electrostatic induction. When fully released and returned to the original state, in (iv), the balanced charge does not generate any current. When a mechanical force is again applied to the device, as seen in (v), a friction charge is again formed on the surface and the principle seen in step (ii) is repeated. 
a

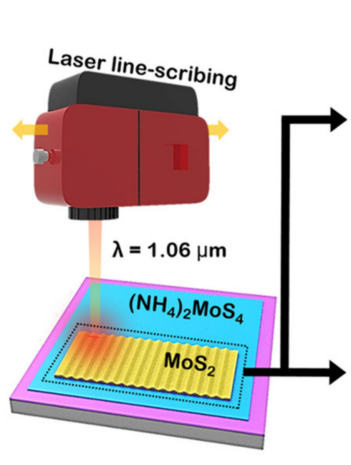

C

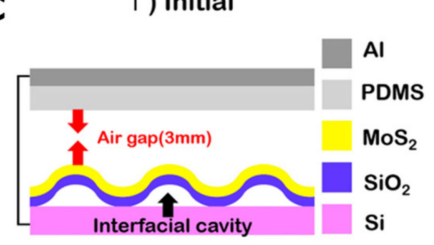

iii) Releasing

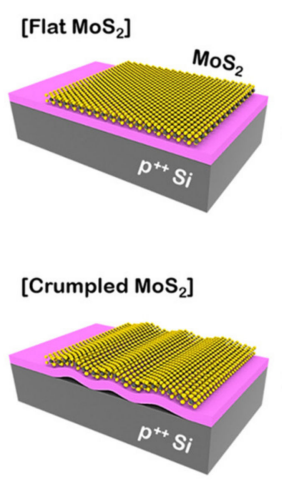

ii) Pressed b

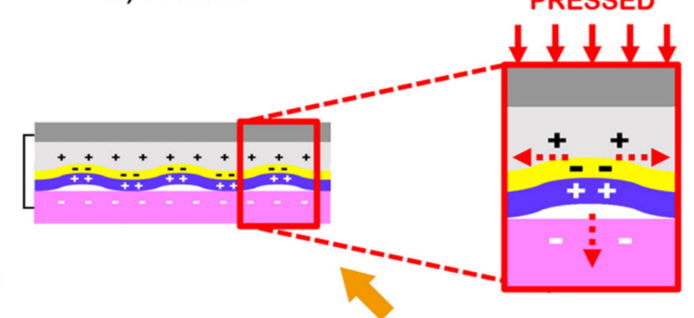

iv) Released

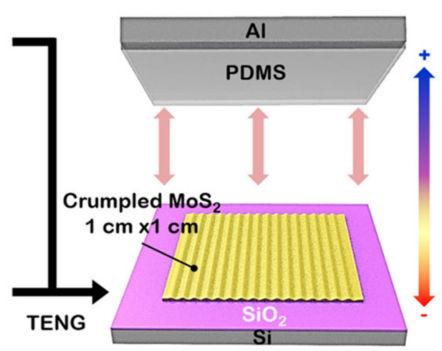

v) Pressing

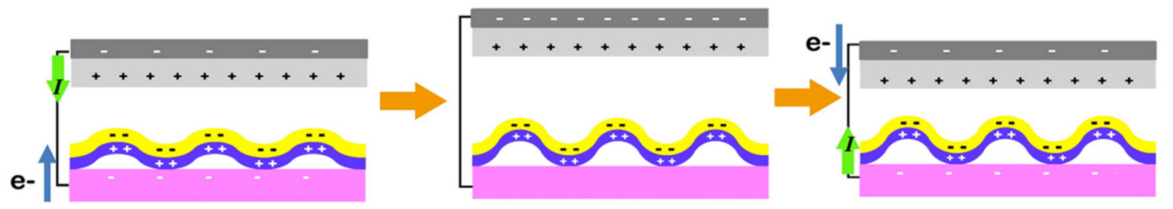

Figure 10. (a) Illustration of laser-induced synthesis of $2 \mathrm{D} \mathrm{MoS}_{2}$ on the $\mathrm{SiO}_{2} / \mathrm{Si}$ wafer. (b) Illustration

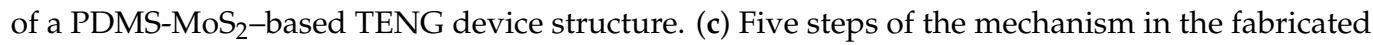
TENG device. Reproduced with permission from [74], Copyright 2020, Elsevier.

Seol et al. investigated the triboelectric charging behaviors of various 2D materials to understand separate working mechanisms in TENG. They used commercially available 2D nanomaterials, such as $\mathrm{MoS}_{2}, \mathrm{MoSe}_{2}, \mathrm{WS}_{2}, \mathrm{WSe}_{2}, \mathrm{GR}$, and graphene oxide (GO), which were chemically exfoliated from their bulk materials. Then, a thin film of the 2D nanomaterials was prepared using a vacuum filtration method. Figure 11a is a schematic showing the $\mathrm{MoS}_{2}$-nylon TENG device structure consisting of two pairs of $\mathrm{Cu}$ electrodes. Figure $11 \mathrm{~b}$ shows the output voltage characteristics for the $\mathrm{MoS}_{2}$-nylon TENG. There are typically four distinctive parts in the output characteristics, as shown in Figure 11c. The designated numbers (1)-(4) in the plot of Figure 11c matches the released, pressing, pressed, and releasing steps depicted in Figure 11a. In particular, a charge transfer is induced by physical contact between $\mathrm{MoS}_{2}$ and the nylon (see Figure 11(a(3))). After contact, releasing the two materials induces a reversed electrical current in the external circuit between the two $\mathrm{Cu}$ electrodes. Thus, a negative voltage is observed at (4) in Figure 11c. It determines that $\mathrm{MoS}_{2}$ is negatively electrified when contacted by nylon. Then, a positively induced voltage is observed in the $\mathrm{MoS}_{2}$-nylon TENG device, which indicates that the transferred electrons transport in the reverse direction through the external load (see Figure 11(a(2))). In a similar manner, various TENGs were fabricated using $\mathrm{MoS}_{2}$ and commercially available polymer films, as shown in Figure 11d. From Figure $11 \mathrm{~d}$, it is possible to quantify the degree of triboelectric behavior, depending on which base material is used. The output value is measured during repeated press and release functions. Interestingly, TENGs with $\mathrm{MoS}_{2}$-PTFE films show negative output during the press, but positive output during the release. This shows that $\mathrm{MoS}_{2}$ becomes anodized after contact with PTFE. Conversely, PDMS, PC, PET, and mica exhibit cathodic properties. By documenting this, we can summarize the triboelectric series as shown 
in Figure 11e for several two-dimensional materials and polymer films. $\mathrm{MoS}_{2}$ had the most negative triboelectric charging performances than other $2 \mathrm{D}$ materials $\left(\mathrm{MoS}_{2}, \mathrm{MoSe}_{2}\right.$, graphene, graphene oxide, $\mathrm{WS}_{2}, \mathrm{WSe}_{2}$ ) and all the $2 \mathrm{D}$ materials were placed at the negative side of the triboelectric series, compared to the studied polymeric films (PC, PET, mica, and nylon). Importantly, the synthetic method and the thickness of $\mathrm{MoS}_{2}$ are barely associated with charging polarities. Instead, the charging characteristics of the triboelectric effect in $\mathrm{MoS}_{2}$ could be strongly affected by chemical doping to modify the work.

a

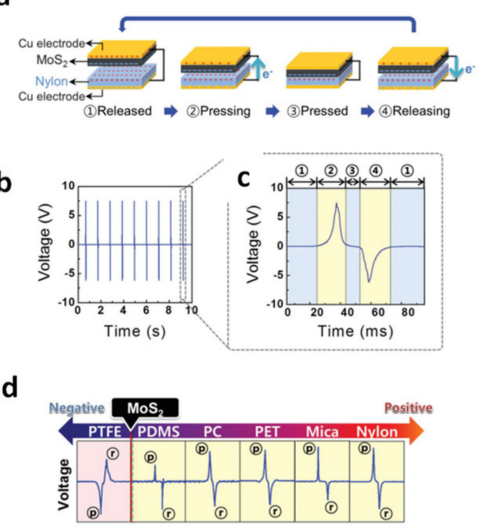

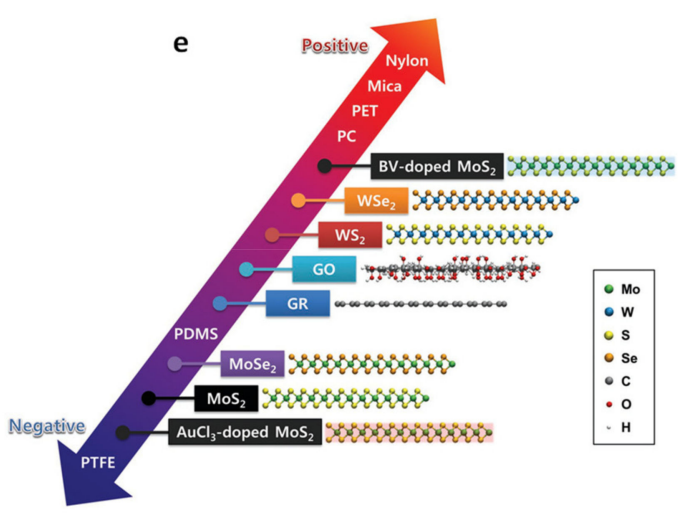

Figure 11. Triboelectric behavior of $\mathrm{MoS}_{2}$ and several polymer films: (a) the device structure and working principle of $\mathrm{MoS}_{2}$-nylon TENG, (b) the output voltage signal, and (c) its output voltage signal in one cycle. (d) Triboelectric behavior of various polymer film-MoS 2 combinations. (e) The modified triboelectric series with 2D materials. Reproduced with permission from [75]. Copyright 2018, John Wiley and Sons.

\subsection{Self-Powered Thermoelectric Devices}

Heat and electricity are very intimate forms of energy in daily life. However, the applicability of heat and electricity is quite different. Heat can be found everywhere around us, but it is difficult to change it to a more useful form in a thermodynamic way, whereas electricity is more practical for powering electrical appliances, but electricity is not ubiquitous and must be stored. Thermoelectrics provides a direct solution for converting heat to electricity in an eco-friendly way [50]. For example, a thermocouple can generate electrical current to operate processes directly, without the need for extra circuitry and power sources. Thus, the power from a thermocouple can trigger a valve when a temperature difference arises. In a thermocouple, continuous heat from the hot side generates the electrical energy to maintain the electric potential between hot and cold sides, in which the Peltier effect dominates to drive the electrical charge in the circuitry. A thermal flow occurs to lower the temperature of the hot side, thereby causing electrical flow. Therefore, it is important to drive the device to allow continuous heat flow. A thermoelectric device produces a voltage when there is a temperature difference between the hot and cold sides. On the other hand, when a voltage is applied to the thermocouple, it produces a temperature difference. Figure $12 \mathrm{a}$ is a schematic of a typical thermoelectric sensor. At the macroscopic scale, an applied temperature gradient force charges the carrier in the material, diffusing it from the hot to the cold side, which generates electricity in the sensor circuitry. In addition, an exact temperature, or a change in the temperature of objects, can be measured by reading electrical current amperage. The term thermoelectric effect implies three effects separately: the Seebeck effect, the Peltier effect, and the Thomson effect. The Seebeck effect is a potential difference generated in a closed circuit due to a temperature difference. The Peltier effect is a temperature difference that occurs when one side is exothermic and the other side is endothermic when a potential difference is applied. The Thomson effect refers to an exothermic or endothermic phenomenon that occurs when an electric current flows and there is a partial temperature difference in the same metal. 
For example, the negative Thomson effect is when current flows from a low-temperature region to a high-temperature region, and it becomes endothermic (for example, $\mathrm{Pt}, \mathrm{Ni}$, or $\mathrm{Fe}$ ). The positive Thomson effect is when a current flows from a high temperature region to a low-temperature region, and it becomes exothermic (for example, $\mathrm{Cu}$ and $\mathrm{Sb}$ ). Thermoelectrics is currently looking for specialized niche applications in the 21st century. In particular, although the current research trend places a lower priority on efficiency than energy availability and reliability, research on availability and reliability is required. Specifically, it is necessary to develop high-performance materials for the wide application of thermoelectric devices in the future. Because thermoelectric materials generate electricity with a change in temperature, the dimensionless figure of merit, $\mathrm{ZT}=\alpha^{2} \mathrm{~T} \rho^{-1} \mathcal{K}^{-1}$, can be calculated from the Seebeck coefficient $(\alpha)$, electrical resistivity $(\rho)$, and thermal conductivity $(\kappa)$. In this regard, for manufacturing high-performance thermoelectric devices, high ZT is an important criterion for determining material performance. Therefore, in order to improve ZT, it is necessary to optimize electrical resistance, the Seebeck coefficient, and thermal conductivity. Achieving high material performance in terms of the microscopic atomic-scale level comes with delicate balances between the phase stability and instability, structural order and disorder, bond covalence and ionicity, band convergence and splitting, transversal and local electronic states, and trade-offs between carrier mobility and effective mass (Figure 12b).
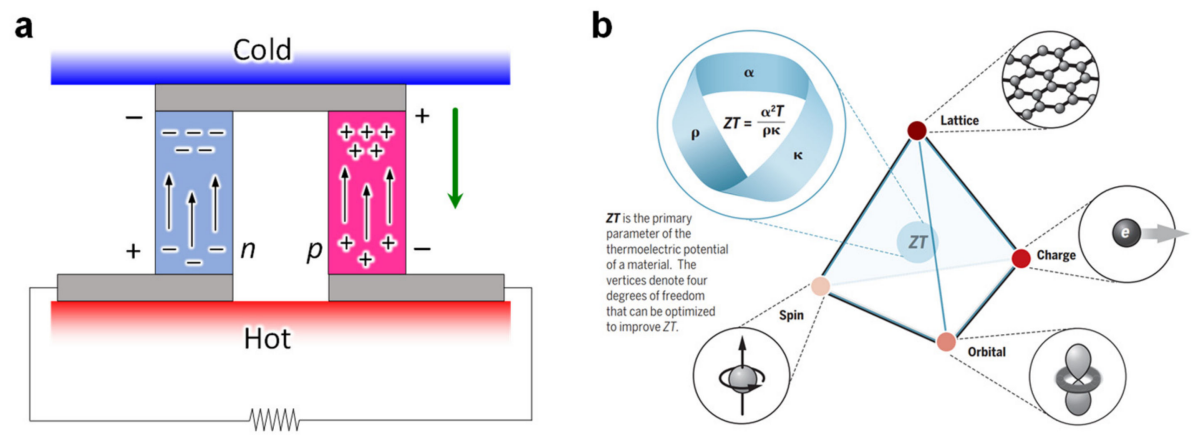

Figure 12. (a) A schematic of a thermoelectric sensor. (b) Thermoelectric materials research is a multidisciplinary study involving charges, spins, orbitals, and lattice degrees of freedom in matter. Studies into electrical resistivity $(\rho)$, the Seebeck coefficient $(\alpha)$, and thermal conductivity $(\kappa)$ should be conducted simultaneously. Reproduced with permission from [50], Copyright 2017, American Association of the Advancement of Science.

Oh et al. fabricated a two-dimensional nanosheet-based flexible thermoelectric device that can obtain energy at room temperature from body temperature [76]. Through chemical exfoliation in a solution process method, a large amount of TMD nanosheets that can be used in the manufacture of thermoelectric devices were synthesized. Figure 13a-d are illustrations showing the process such as the preparation of chemically exfoliated TMD nanosheet films to fabricate wearable thermoelectric generators. A solution can be prepared that separates the bulk TMD material into single/several layers of 2D nanosheets through a chemical exfoliation process. There are two steps in this process. The first step is to insert $\mathrm{Li}$ ions into the interlayers of the bulk TMD. n-Butyl lithium (Bu-Li) molecules migrate into octahedral sites per interlayer of the bulk TMD, and $\mathrm{Li}$ atoms and TMD meet to form a LixTMD compound from their high chemical affinity. Next, Li ions of the LixTMD compound react with DI water to form $\mathrm{LiOH}$, and the lamellar TMD is peeled off. This reaction causes the interlayer space of the TMD to expand, weakening the van der Waals force, and can easily be exfoliated by sonication. This chemically peeled TMD solution can be vacuum filtered through a membrane to produce the desired TMD thin film (Figure 13b). The TMD film produced in this way can be patterned into the desired shape, and TMDPDMS thermoelectric devices can be fabricated through the contact printing method (Figure 13c,d). To fabricate a thermoelectric generator, n-type and p-type TMD films must 
be prepared and fabricated on a PDMS substrate. Figure 13e shows $\mathrm{WS}_{2}$ (n-type) and $\mathrm{NbSe}_{2}$ nanosheet (p-type) solutions. The $\mathrm{WS}_{2}$ film used as the n-type thermoelectric film exhibits the highest Seebeck coefficient of approximately -70 to $-75 \mu \mathrm{Vk}^{-1}$ (Figure 13f) and high conductivity (approximately 1.0 to $1.4 \times 10^{3} \mathrm{Sm}^{-1}$ ) at room temperature. On the other hand, p-type $\mathrm{NbSe}_{2}$ shows about $+12 \sim+14 \mu \mathrm{Vk}^{-1}$ for the Seebeck coefficient, and about $150 \sim 170 \times 10^{3} \mathrm{Sm}^{-1}$ in conductivity, as shown in Figure 13g. A wearable thermoelectric generator consisting of those 2D TMD nanosheets can be used to generate electric energy in a self-powered way from the human wrist through the thermoelectric generator.

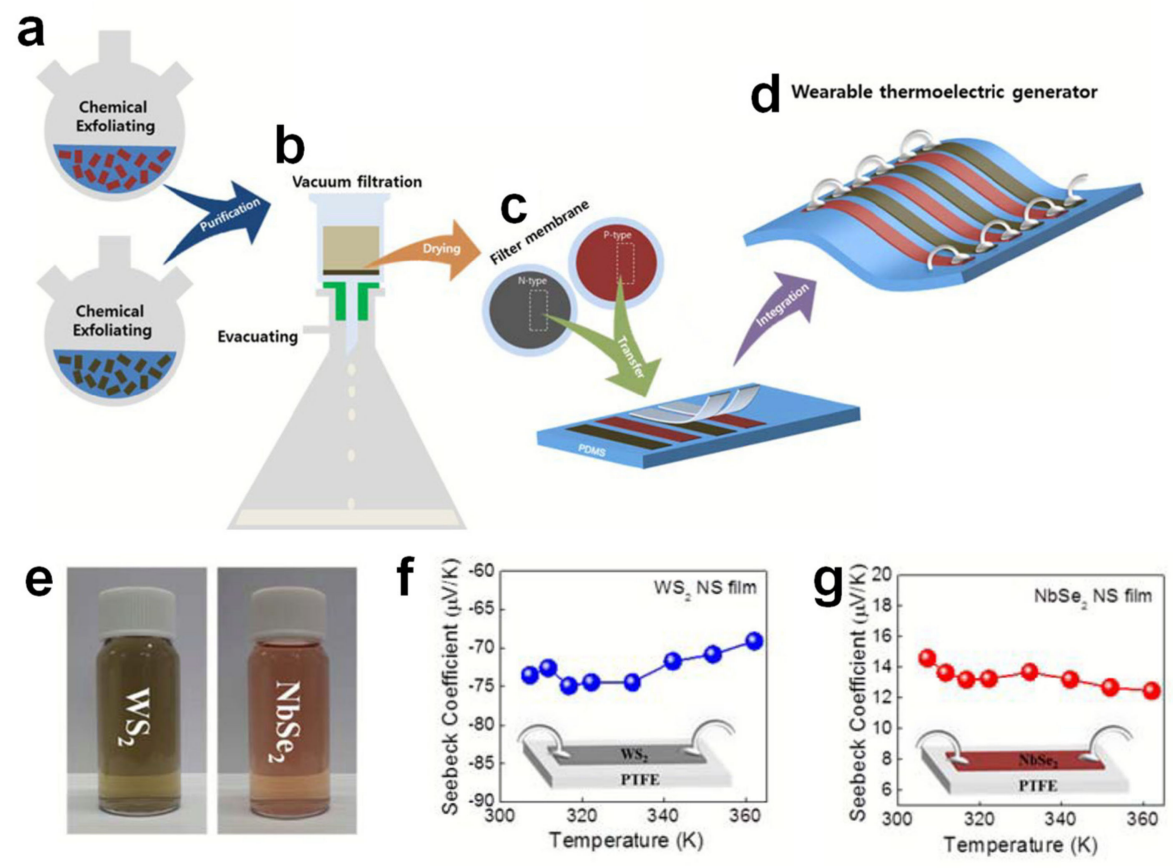

Figure 13. Chemically exfoliating TMD nanosheets and manufacturing a wearable thermoelectric generator using TMD nanosheets: (a) preparation of chemically exfoliated TMD, (b) vacuum filtration of TMD nanosheets using a membrane filter, (c) transfer of a vacuum-filtered TMD nanosheet film to a PDMS substrate using a contact printing method, and (d) the connection between Ag wire and thermoelectric film using $\mathrm{Ag}$ paste. (e) $\mathrm{WS}_{2}$ and $\mathrm{NbSe}_{2}$ dispersed in aqueous solution, With graphical representations of thermoelectric properties from $300 \mathrm{~K}$ to $360 \mathrm{~K}$ for chemically exfoliated (f) $\mathrm{WS}_{2}$ and (g) $\mathrm{NbSe}_{2}$. Reproduced with permission from [76], Copyright 2016, Royal Society of Chemistry.

\subsection{Self-Powered Sensor Integration}

The aforementioned 2D material-based self-powered sensors offer extremely low power consumption, and integrated applications have been developed. A self-powered circuit integration of perovskite cells and $2 \mathrm{D} \mathrm{MoTe}_{2}$ transistors was successfully demonstrated by Im et al. in 2019 [77]. Methylammonium lead iodide perovskite photovoltaic cells connected with $\mathrm{MoTe}_{2}$ transistors provided a dynamic response under a red light pulse at $1 \mathrm{~Hz}$ (Figure 14a,b). Furthermore, they implemented photovoltaic-powered green organic light-emitting diodes (OLEDs), presenting pulsed green light illumination according to white light-source switching behaviors (Figure 14c,d). From remarkable progress in 2D materials-based self-powered sensor integration, a TENG integrated array using MXene was demonstrated by Shan et al. in 2021 [78]. An alternating current electroluminescence (ACEL) array connecting TENG device integrations led to a self-powered pattern display. The flexible and transparent $4 \mathrm{~mm}^{2}$ ACEL display arrays successfully exhibited pattern Z (Figure 14e). 
a

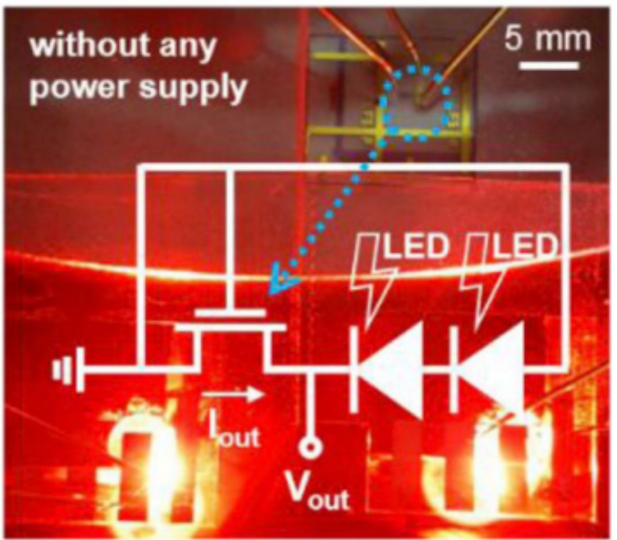

C
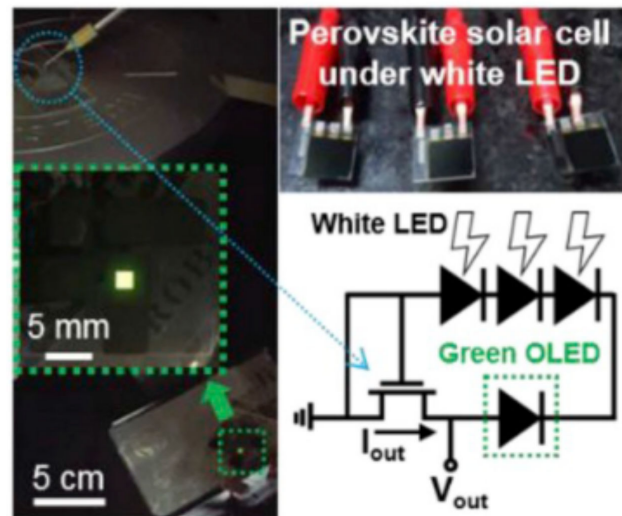

b

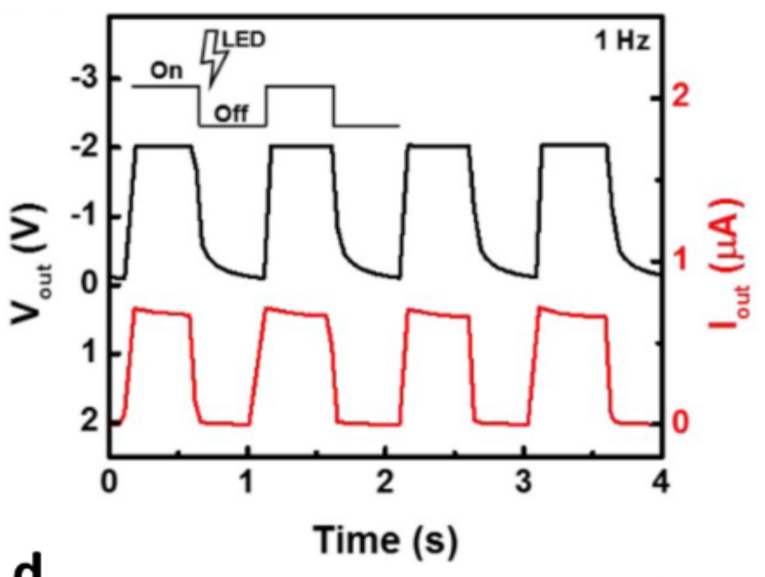

d

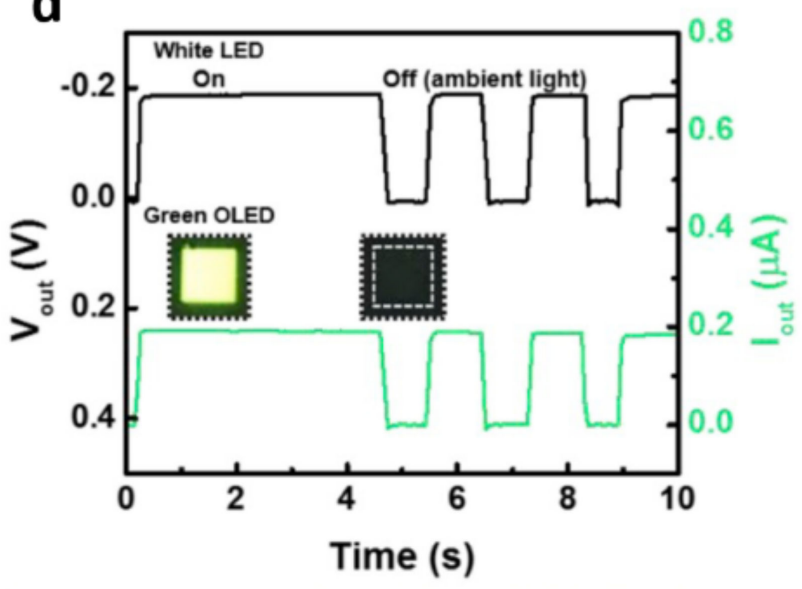

e

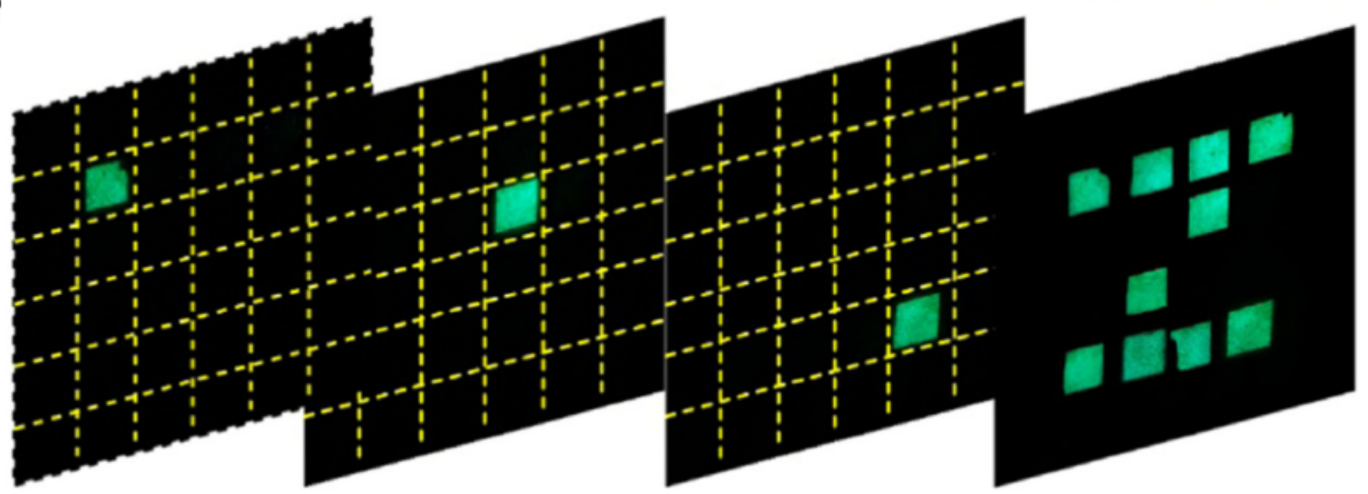

Figure 14. Self-powered integrations: (a) optical image of the $\mathrm{MoTe}_{2}$ transistor and perovskite photovoltaic cells connected in series under red LED illumination, and (b) pulsed measurement of an integrated circuit under a red LED pulse at $1 \mathrm{~Hz}$; (c) integration of green OLEDs combined with perovskite photovoltaic cells and $\mathrm{MoTe}_{2}$ switching transistors, and (d) pulsed measurement of green OLED under white LED switching. Reproduced with permission from [77], Copyright 2019, Elsevier. (e) Pattern array image of MXene-based alternating current electroluminescence integration. Reproduced with permission from [78], Copyright 2021, Elsevier.

In 2021, a 2D metal-organic framework (MOF) and polyvinylidene fluoride (PVDF)based piezoelectric nanogenerators were presented by Sinha and Mandal et al. [79]. In addition to the excellent piezoelectric output at as high a power density as $24 \mu \mathrm{W} \cdot \mathrm{cm}^{-2}$, an acoustoelectric application was demonstrated (Figure 15a). At a $110 \mathrm{~dB}$ acoustic vibration, successful acoustoelectric conversion was performed, exhibiting energy-harvesting behavior from $120 \mathrm{~Hz}$ at $110 \mathrm{~dB}$ for five blue light-emitting diodes. Furthermore, the proposed acoustoelectric conversion was demonstrated with five musical instruments (guitar, 
drum, piano, saxophone, and the tabla) (Figure 15b). As another interesting application, a self-powered stretchable TENG-based touch sensor was presented by Ahn et al. [80]. From atomically thin graphene integrated with a polyethylene terephthalate substrate and a poly-dimethyl siloxane electrification layer, a highly stretchable TENG array was fabricated. Touch-sliding velocity was successfully detected through the graphene-based stretchable TENG sensors, providing self-powered operation without an energy source.

a

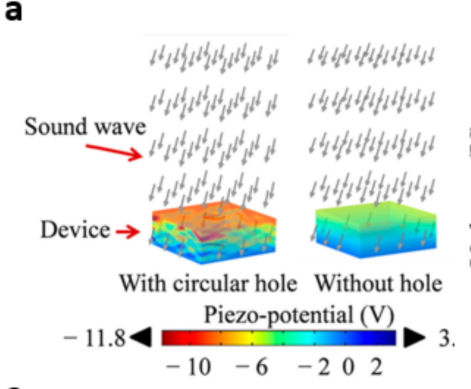

C

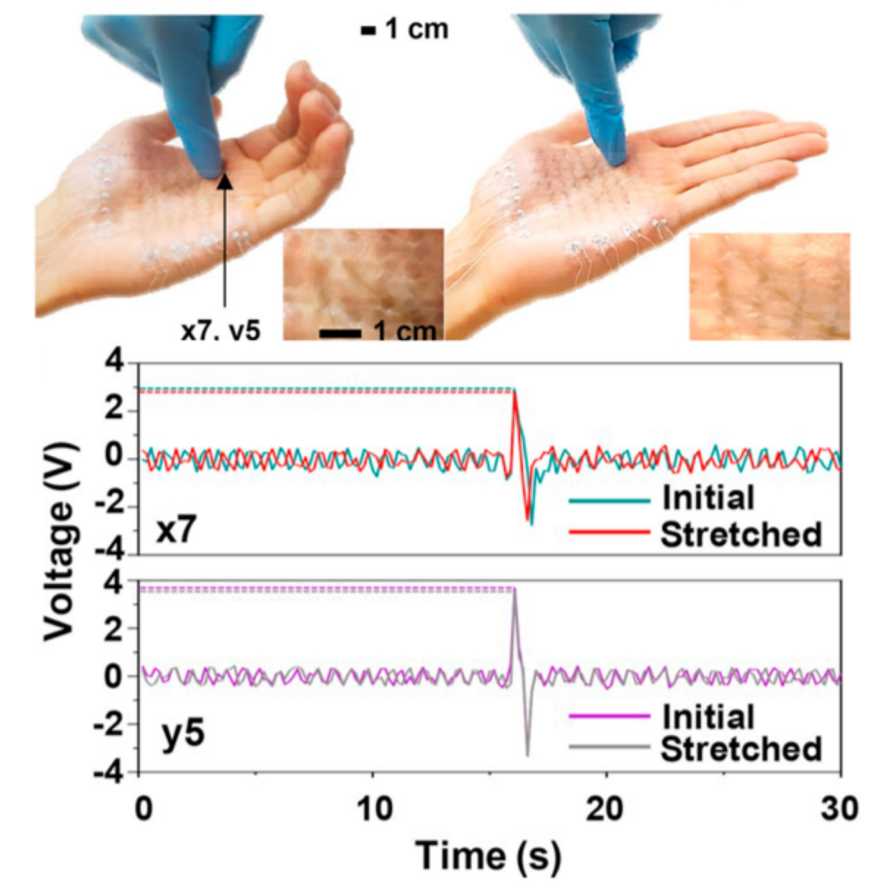

b

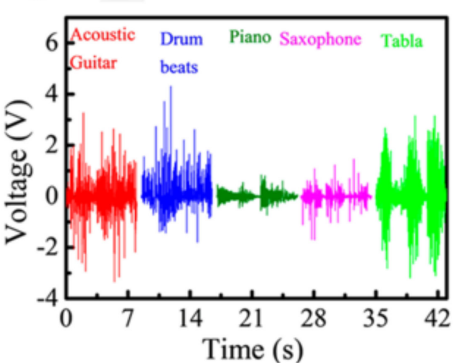

$$
\text { (2) }
$$

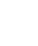


neuromorphic system (Figure 16a-c). In addition, a self-powered nanogenerator-based memristor was proposed [82]. Connecting amorphous carbon nanogenerators to the memristor demonstrated self-powered nanogenerator-memristor cells, exhibiting set/resetbased low resistance-state (LRS) and high resistance-state (HRS) switching behaviors (Figure 16d).

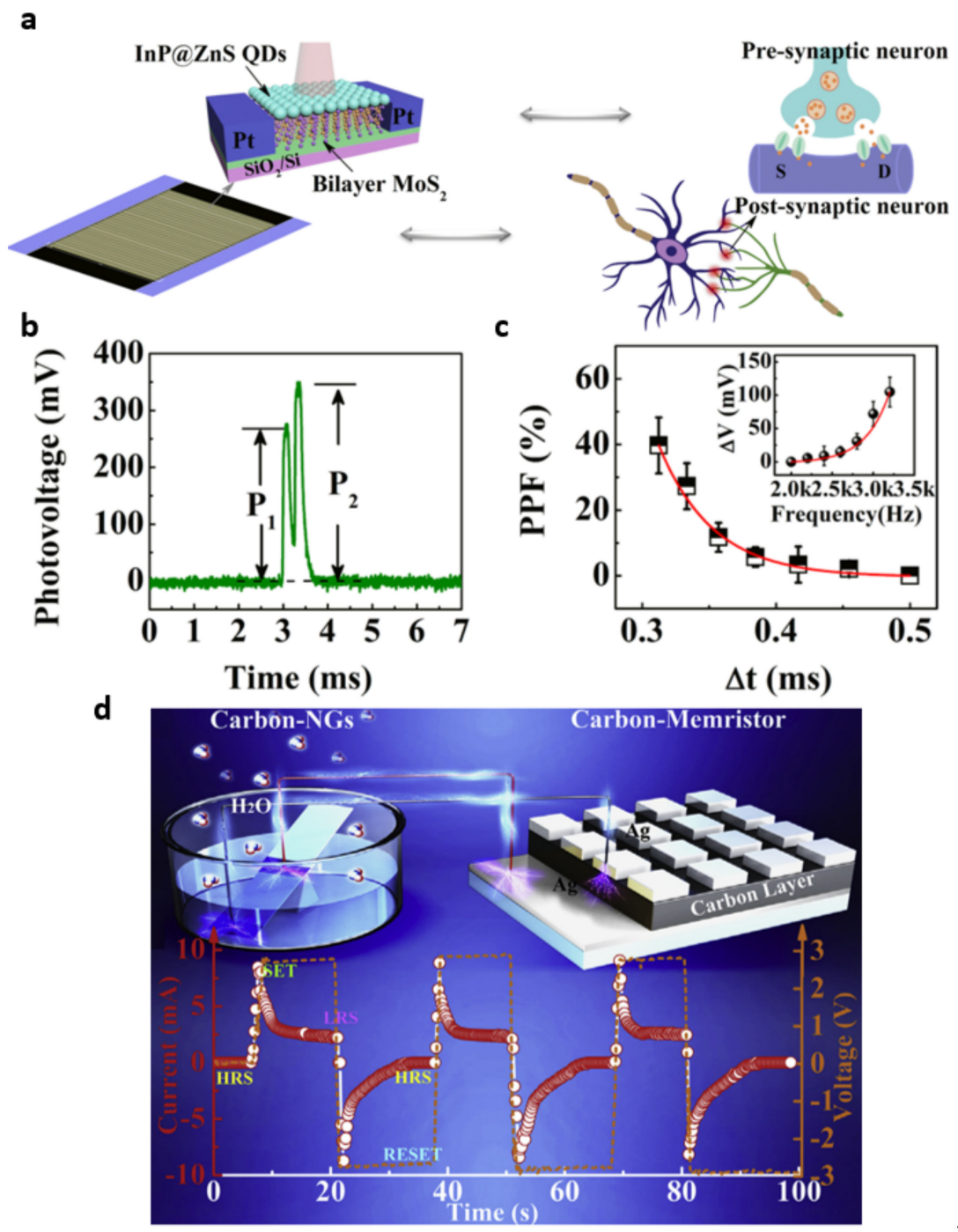

Figure 16. Self-powered neuromorphic systems: (a) the InP@ZnS-MoS 2 hybrid phototransistor and a neural network with a multiple series-parallel synapses schematic, (b) paired-pulse facilitation (PPF) induced by two laser pulses under a $532 \mathrm{~nm}$ laser at $170 \mathrm{~mW} \cdot \mathrm{cm}^{-2}$, and (c) dependence of the PPF on the interval between paired pulses $(\Delta \mathrm{t})$ from a $532 \mathrm{~nm}$ laser at $170 \mathrm{~mW} \cdot \mathrm{cm}^{-2}$; the inset shows the variance of the photovoltage as a function of pulse frequency from 2000 to $3200 \mathrm{~Hz}$. Reproduced with permission from [81], Copyright 2020, American Chemical Society. (d) A self-powered system using carbon-NGs cells connected in series with a memristor. The air velocity is controlled at $\sim 1.0 \mathrm{~ms}^{-1}$. The output current versus the time for the carbon memristor after integrating it with three carbonNGs cells shows the expected voltage output. Reproduced with permission from [82]. Copyright 2019, Elsevier.

\section{Conclusions}

Various examples of self-powered 2D-nanomaterial sensors offer many benefits, as revisited in this paper. However, further advances are required for practical application development. The 2D materials-based sensors still have challenges, and there are many directions for future research. The following requirements should be addressed: (1) develop 
a large-area synthesis method for 2D nanomaterials, (2) establish a reliable phase control engineering process, (3) secure long-term reliability and stability, and (4) implement process development that is compatible with flexible substrates.

First, to realize an integrated, self-powered system at more than a sensor array level, large-area synthesis technology with a high yield must be developed. The current technology status is still implementation at the flake level or the centimeter level containing defects or grain boundaries. To make 2D nanomaterials available for real sensor systems, a uniform and robust materials platform should be developed. Second, the 2D-material phase control process must be further developed. Optoelectrical properties of 2D nanomaterials significantly depend on their crystalline phase, such as $1 \mathrm{~T}, 2 \mathrm{H}$, and $3 \mathrm{R}$. The material needs to be adjusted to make it compatible with the function of each electronic sensor element. Third, in order to gather big data and to be used in the IoT, long-term stability should be secured. Still, previously reported sensor devices have shown a lack of long-term stability evaluation. It is necessary to introduce a new evaluation method to realize a sensor for long-term continuous healthcare monitoring based on self-powered technology. Fourth, mechanical flexibility in plastic substrate process development should be accomplished. For wireless technology and healthcare sensors, wearable devices are essential. To maximize the potential benefits of 2D nanomaterials, compatibility with flexible substrates is needed. As mentioned, self-powered 2D-nanomaterial sensors still have difficulties in the synthesis and fabrication processes, and with reliability. However, the advantages and possibilities from their functionality are expected to help us envision autonomous wearable sensors.

Author Contributions: E.K.L. and H.Y. performed literature research, analysis, and wrote the paper. Both authors have read and agreed to the published version of the manuscript.

Funding: This research was supported by a National Research Foundation of Korea (NRF) grant funded by the Korean Government (MSIT) (NRF-2020R1A2C1101647 and NRF-2020M3A9E4104385).

Conflicts of Interest: The authors declare no conflict of interest.

\section{References}

1. Ornelas, C.D.; Bowman, A.; Walmsley, T.S.; Wang, T.; Andrews, K.; Zhou, Z.; Xu, Y.-Q. Ultrafast Photocurrent Response and High Detectivity in Two-Dimensional MoSe 2 -based Heterojunctions. ACS Appl. Mater. Interfaces 2020, 12, 46476-46482. [CrossRef]

2. Hong, S.; Zagni, N.; Choo, S.; Liu, N.; Baek, S.-H.; Bala, A.; Yoo, H.; Kang, B.H.; Kim, H.J.; Yun, H.-J.; et al. Highly sensitive active pixel image sensor array driven by large-area bilayer $\mathrm{MoS}_{2}$ transistor circuitry. Nat. Commun. 2021, 12, 3559. [CrossRef] [PubMed]

3. Jansen-van Vuuren, R.D.; Nunzi, J.-M.; Givigi, S.N. Frontiers in Photosensor Materials and Designs for New Image Sensor Applications. IEEE Sens. J. 2020, 21, 10. [CrossRef]

4. Kim, S.; Hong, S.; Yoo, H. Location-dependent multi-parameter detection behaviors using hetero-interfaced organic anti-ambipolar phototransistors. Sens. Actuators A Phys. 2021, 330, 112888. [CrossRef]

5. Wang, Z.; Zhi, M.; Xu, M.; Guo, C.; Man, Z.; Zhang, Z.; Qiang, L.; Lv, Y.; Zhao, W.; Yan, J.; et al. Ultrasensitive $\mathrm{NO}_{2}$ gas sensor based on $\mathrm{Sb}$-doped $\mathrm{SnO}_{2}$ covered $\mathrm{ZnO}$ nano-heterojunction. J. Mater. Sci. 2021, 56, 7348-7356. [CrossRef]

6. Xie, J.; Zhang, L.; Liu, B.; Bai, P.; Chenjie, W.; Jiake, X.; Wang, H. Highly Selective Gas Sensor Based on Hydrophobic Silica Decorated with Trimethoxyoctadecylsilane. ACS Appl. Mater. Interfaces 2020, 13, 1956-1966. [CrossRef] [PubMed]

7. Nakajima, T.; Tsuchiya, T. Ultrathin Highly Flexible Featherweight Ceramic Temperature Sensor Arrays. ACS Appl. Mater. Interfaces 2020, 12, 36600-36608. [CrossRef] [PubMed]

8. Liu, B.; Luo, J.; Liu, S.; Chen, Y.; Huang, B.; Liao, C.; Wang, Y. A Probe-Shaped Sensor with FBG and Fiber-Tip Bubble for Pressure and Temperature Sensing. Photonic Sens. 2021, 1-7. [CrossRef]

9. Song, X.; Liu, H.; Fang, Y.; Zhao, C.; Ziqiang, Q.; Qiu, W.; Tu, L.-C. An Integrated Gold-Film Temperature Sensor for In Situ Temperature Measurement of a High-Precision MEMS Accelerometer. Sensors 2020, 20, 3652. [CrossRef]

10. Chen, Y.; Wu, W.; Yu, J.; Wang, Y.; Zhu, J.; Hu, Z. Mechanical strong stretchable conductive multi-stimuli-responsive nanocomposite double network hydrogel as biosensor and actuator. J. Biomater. Sci. Polym. Ed. 2020, 31, 1-23. [CrossRef]

11. Molinnus, D.; Beging, S.; Lowis, C.; Schöning, M.J. Towards a Multi-Enzyme Capacitive Field-Effect Biosensor by Comparative Study of Drop-Coating and Nano-Spotting Technique. Sensors 2020, 20, 4924. [CrossRef]

12. Zhao, M.-H.; Cui, L.; Sun, B.; Wang, Q.; Zhang, C.-Y. Low-background electrochemical biosensor for one-step detection of base excision repair enzyme. Biosens. Bioelectron. 2019, 150, 111865. [CrossRef]

13. Cao, Q.; Liang, B.; Yu, C.; Fang, L.; Tu, T.; Wei, J.; Ye, X. High accuracy determination of multi metabolite by an origami-based coulometric electrochemical biosensor. J. Electroanal. Chem. 2020, 873, 114358. [CrossRef] 
14. Liu, S.-Y.; Lu, J.; Shieh, H.-P.D. Influence of Permittivity on the Sensitivity of Porous Elastomer-Based Capacitive Pressure Sensors. IEEE Sens. J. 2018, 18, 1870-1876. [CrossRef]

15. Yu, Y.; Zhang, Y.; Jin, L.; Chen, Z.; Li, Y.; Li, Q.; Cao, M.; Che, Y.; Yang, J.; Yao, J. A Fast Response-Recovery 3D Graphene Foam Humidity Sensor for User Interaction. Sensors 2018, 18, 4337. [CrossRef]

16. Qiang, L.; Gao, L.; Su, X.; Zhou, F.; Guotao, D. Interfacial self-assembly of CoPc thin films with their high sensing use as $\mathrm{NO}_{2}$ sensors. Mater. Chem. Phys. 2019, 234, 94-101. [CrossRef]

17. Lin, P.-S.; Shen, T.-W.; Chan, K.-C.; Fang, W. CMOS MEMS Thermoelectric Infrared Sensor with Plasmonic Metamaterial Absorber for Selective Wavelength Absorption and Responsivity Enhancement. IEEE Sens. J. 2020, 20, 11105-11114. [CrossRef]

18. Son, H.; Cho, H.; Koo, J.; Ji, Y.; Kim, B.; Park, H.-J.; Sim, J.-Y. A Low-Power Wide Dynamic-Range Current Readout Circuit for Ion-Sensitive FET Sensors. IEEE Trans. Biomed. Circuits Syst. 2017, 11, 523-533. [CrossRef] [PubMed]

19. Palacio, F.; Fonollosa, J.; Burgués, J.; Gómez, J.M.; Marco, S. Pulsed-temperature metal oxide gas sensors for microwatt power consumption. IEEE Access 2020, 8, 70938-70946. [CrossRef]

20. Kalaivaani, P.T.; Krishnamoorthi, R. Design and implementation of low power bio signal sensors for wireless body sensing network applications. Microprocess. Microsyst. 2020, 79, 103271. [CrossRef]

21. Kim, J.-H.; Mirzaei, A.; Kim, H.W.; Kim, S.S. Realization of Au-decorated WS2 nanosheets as low power-consumption and selective gas sensors. Sens. Actuators B Chem. 2019, 296, 126659. [CrossRef]

22. Kim, J.-H.; Mirzaei, A.; Kim, H.W.; Kim, S.S. Low power-consumption CO gas sensors based on Au-functionalized $\mathrm{SnO}_{2}-\mathrm{ZnO}$ core-shell nanowires. Sens. Actuators B Chem. 2018, 267, 597-607. [CrossRef]

23. Minfeng, T.; Qihui, G.; Wu, X.; Zeng, X.; Zhang, Z.; Yuan, Y. A high-efficiency multidirectional wind energy harvester based on impact effect for self-powered wireless sensors in the grid. Smart Mater. Struct. 2019, 28, 115022. [CrossRef]

24. Salehi, H.; Burgueño, R.; Chakrabartty, S.; Lajnef, N.; Alavi, A.H. A comprehensive review of self-powered sensors in civil infrastructure: State-of-the-art and future research trends. Eng. Struct. 2021, 234, 111963. [CrossRef]

25. Li, M.; Jiaxin, C.; Zhong, W.; Luo, M.; Wen, W.; Qing, X.; Ying, L.; Liu, Q.; Liu, K.; Wang, Y.; et al. Large-Area, Wearable, Self-Powered Pressure-Temperature Sensor Based on 3D Thermoelectric Spacer Fabric. ACS Sens. 2020, 5, 2545-2554. [CrossRef]

26. Cheng, P.; Sun, X.; Sun, M.; Zhang, C.; Guo, H.; Shi, J.; Zhang, Y.; Liu, Y.; Wang, J.; Wen, Z. Self-Powered Active Spherical Triboelectric Sensor for Fluid Velocity Detection. IEEE Trans. Nanotechnol. 2020, 19, 230-235. [CrossRef]

27. Jiang, M.; Lu, Y.; Zhu, Z.; Jia, W. Advances in Smart Sensing and Medical Electronics by Self-Powered Sensors Based on Triboelectric Nanogenerators. Micromachines 2021, 12, 698. [CrossRef]

28. Ge, R.; Wu, X.; Liang, L.; Hus, S.M.; Gu, Y.; Okogbue, E.; Chou, H.; Shi, J.; Zhang, Y.; Banerjee, S.K.; et al. A Library of Atomically Thin 2D Materials Featuring the Conductive-Point Resistive Switching Phenomenon. Adv. Mater. 2021, 33, 2007792. [CrossRef]

29. Wang, S.; Ning, H.; Hu, N.; Liu, Y.; Liu, F.; Zou, R.; Huang, K.; Wu, X.; Weng, S.; Alamusi, N. Environmentally-Friendly and Multifunctional Graphene-Silk Fabric Strain Sensor for Human-Motion Detection. Adv. Mater. Interfaces 2019, 7, 1901507. [CrossRef]

30. Han, D.-D.; Zhang, Y.-L.; Ma, J.-N.; Liu, Y.; Mao, J.-W.; Han, C.-H.; Jiang, K.; Zhao, H.; Zhang, T.; Xu, H.; et al. Sunlight-Reduced Graphene Oxides as Sensitive Moisture Sensors for Smart Device Design. Adv. Mater. Technol. 2017, 2, 1700045. [CrossRef]

31. Sajid, M.; Kim, H.B.; Lim, J.H.; Choi, K.H. Liquid assisted exfoliation of 2D hBN flakes and their dispersion in PEO to fabricate highly specific and stable linear humidity sensors. J. Mater. Chem. C 2018, 6, 1421-1432. [CrossRef]

32. Goel, N.; Kumar, M. Recent advances in ultrathin 2D hexagonal boron nitride based gas sensors. J. Mater. Chem. C 2021, 9 , 1537-1549. [CrossRef]

33. Li, B.L.; Wang, J.; Zou, H.L.; Garaj, S.; Lim, C.T.; Xie, J.; Li, N.B.; Leong, D.T. Low-Dimensional Transition Metal Dichalcogenide Nanostructures Based Sensors. Adv. Funct. Mater. 2016, 26, 7034-7056. [CrossRef]

34. Feng, S.; Lin, Z.; Gan, X.; Lv, R.; Terrones, M. Doping two-dimensional materials: Ultra-sensitive sensors, band gap tuning and ferromagnetic monolayers. Nanoscale Horiz. 2017, 2, 72-80. [CrossRef]

35. Yoo, H.; Hong, S.; Moon, H.; On, S.; Ahn, H.; Lee, H.-K.; Kim, S.; Hong, Y.K.; Kim, J.-J. Chemical Doping Effects on CVD-Grown Multilayer MoSe2 Transistor. Adv. Electron. Mater. 2018, 4, 1700639. [CrossRef]

36. Hong, S.; Yoo, H. Robust molybdenum diselenide ambipolar transistors with fluoropolymer interfacial layer and their application to complementary inverter circuits. J. Alloys Compd. 2021, 868, 159212. [CrossRef]

37. Kim, J.; Lee, E.; Bhoyate, S.; An, T.K. Stable and High-Performance Piezoelectric Sensor via CVD Grown WS 2 . Nanotechnology 2020, 31, 445203. [CrossRef] [PubMed]

38. Luo, X.; Andrews, K.; Wang, T.; Bowman, A.; Zhou, Z.; Xu, Y.-Q. Reversible photo-induced doping in WSe ${ }_{2}$ field effect transistors. Nanoscale 2019, 11, 7358-7363. [CrossRef]

39. Kuiri, M.; Chakraborty, B.; Paul, A.K.; Das, S.; Sood, A.K.; Das, A. Enhancing photoresponsivity using MoTe 2 -graphene vertical heterostructures. Appl. Phys. Lett. 2016, 108, 063506. [CrossRef]

40. Hu, R.-X.; Ma, X.-L.; An, C.-H.; Liu, J. Visible-to-near-infrared photodetector based on graphene-MoTe 2 -graphere heterostructure. Chin. Phys. B 2019, 28, 117802. [CrossRef]

41. Xu, Y.; Liu, C.; Guo, C.; Yu, Q.; Guo, W.; Lu, W.; Chen, X.; Wang, L.; Zhang, K. High performance near infrared photodetector based on in-plane black phosphorus p-n homojunction. Nano Energy 2020, 70, 104518. [CrossRef]

42. Kang, D.-H.; Jeon, M.H.; Jang, S.K.; Choi, W.-Y.; Kim, K.N.; Kim, J.; Lee, S.; Yeom, G.Y.; Park, J.-H. Self-Assembled Layer (SAL)-Based Doping on Black Phosphorus (BP) Transistor and Photodetector. ACS Photonics 2017, 4, 1822-1830. [CrossRef] 
43. Han, S.; Zhang, H.; Lu, Y.; Xu, W.; Fang, M.; Liu, W.; Cao, P.; Zhu, D. Self-Powered Au/MgZnO/Nanolayered Ga-Doped ZnO/In Metal-Insulator-Semiconductor UV Detector with High Internal Gain at Deep UV Light under Low Voltage. ACS Appl. Nano Mater. 2019, 3, 120-130. [CrossRef]

44. Foisal, A.R.M.; Qamar, A.; Nguyen, T.; Dinh, T.; Phan, H.-P.; Nguyen, H.; Duran, P.G.; Streed, E.; Dao, D.V. Ultra-sensitive self-powered position-sensitive detector based on horizontally-aligned double 3C-SiC/Si heterostructures. Nano Energy 2021, 79, 105494. [CrossRef]

45. Xu, Q.; Zhang, B.; Nie, J.; Zhang, H.; Ouyang, X.; Liu, J.; Liu, Y. Self-powered X-ray detector based on methylammonium lead tribromide single crystals. J. Alloys Compd. 2021, 859, 157826. [CrossRef]

46. Wang, Y.; Wu, H.; Xu, L.; Zhang, H.; Yang, Y.; Wang, Z.L. Hierarchically patterned self-powered sensors for multifunctional tactile sensing. Sci. Adv. 2020, 6, eabb9083. [CrossRef]

47. Wu, Z.; Cheng, T.; Wang, Z.L. Self-Powered Sensors and Systems Based on Nanogenerators. Sensors 2020, 20, 2925. [CrossRef] [PubMed]

48. Bowen, C.R.; Kim, H.A.; Weaver, P.M.; Dunn, S. Piezoelectric and ferroelectric materials and structures for energy harvesting applications. Energy Environ. Sci. 2014, 7, 25-44. [CrossRef]

49. Liu, Y.; Ping, J.; Ying, Y. Recent Progress in 2D-Nanomaterial-Based Triboelectric Nanogenerators. Adv. Funct. Mater. 2021, 31, 2009994. [CrossRef]

50. He, J.; Tritt, T.M. Advances in thermoelectric materials research: Looking back and moving forward. Science 2017, 357 , eaak9997. [CrossRef] [PubMed]

51. Geim, A.K.; Novoselov, K.S. The rise of graphene. Nat. Mater. 2007, 6, 183-191. [CrossRef]

52. Qian, Z.; Jiao, L.; Xie, L. Phase Engineering of Two-Dimensional Transition Metal Dichalcogenides. Chin. J. Chem. 2020, 38, 753-760. [CrossRef]

53. Chen, H.; Tang, Y.; Jiang, T.; Li, G. Nonlinear Nanophotonics with 2D Transition Metal Dichalcogenides. In Comprehensive Nanoscience and Nanotechnology; Academic Press: Cambridge, MA, USA, 2019; pp. 305-318. [CrossRef]

54. Ansari, N.; Ghorbani, F. Light absorption optimization in two-dimensional transition metal dichalcogenide van der Waals heterostructures. J. Opt. Soc. Am. B 2018, 35, 1179-1185. [CrossRef]

55. Ahmed, S.; Yi, J. Two-Dimensional Transition Metal Dichalcogenides and Their Charge Carrier Mobilities in Field-Effect Transistors. Nano-Micro Lett. 2017, 9, 50. [CrossRef]

56. Voshell, A.; Terrones, M.; Rana, M. Thermal and Photo Sensing Capabilities of Mono- and Few-Layer Thick Transition Metal Dichalcogenides. Micromachines 2020, 11, 693. [CrossRef]

57. Wang, F.; Wang, Z.; Yin, L.; Cheng, R.; Wang, J.; Wen, Y.; Shifa, T.A.; Wang, F.; Zhang, Y.; Zhan, X.; et al. 2D library beyond graphene and transition metal dichalcogenides: A focus on photodetection. Chem. Soc. Rev. 2018, 47, 6296-6341. [CrossRef]

58. Wang, H.; Wang, X.; Chen, Y.; Zhang, S.; Jiang, W.; Zhang, X.; Qin, J.; Wang, J.; Li, X.; Pan, Y.; et al. Extremely Low Dark Current MoS2 Photodetector via 2D Halide Perovskite as the Electron Reservoir. Adv. Opt. Mater. 2020, 8, 1901402. [CrossRef]

59. Peumans, P.; Yakimov, A.; Forrest, S.R. Small molecular weight organic thin-film photodetectors and solar cells. J. Appl. Phys. 2003, 93, 3693-3723. [CrossRef]

60. Baeg, K.J.; Binda, M.; Natali, D.; Caironi, M.; Noh, Y.Y. Organic light detectors: Photodiodes and phototransistors. Adv. Mater. 2013, 25, 4267-4295. [CrossRef]

61. Jin, W.; Ye, Y.; Gan, L.; Yu, B.; Wu, P.; Dai, Y.; Meng, H.; Guo, X.; Dai, L. Self-powered high performance photodetectors based on CdSe nanobelt/graphene Schottky junctions. J. Mater. Chem. 2012, 22, 2863-2867. [CrossRef]

62. Yang, S.; Wang, C.; Ataca, C.; Li, Y.; Chen, H.; Cai, H.; Suslu, A.; Grossman, J.C.; Jiang, C.; Liu, Q.; et al. Self-Driven Photodetector and Ambipolar Transistor in Atomically Thin GaTe-MoS2 p-n vdW Heterostructure. ACS Appl. Mater. Interfaces 2016, 8, $2533-2539$. [CrossRef]

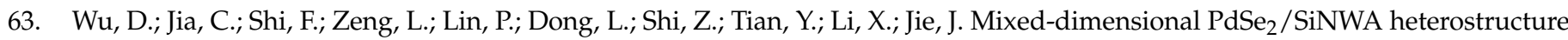
based photovoltaic detectors for self-driven, broadband photodetection, infrared imaging and humidity sensing. J. Mater. Chem. A 2020, 8, 3632-3642. [CrossRef]

64. Wu, J.; Yang, Z.W.; Qiu, C.; Zhang, Y.-J.; Wu, Z.; Yang, J.-L.; Lu, Y.; Li, J.-F.; Yang, D.; Hao, R.; et al. Enhanced performance of a graphene/GaAs self-driven near-infrared photodetector with upconversion nanoparticles. Nanoscale 2018, 10, 8023-8030. [CrossRef]

65. Zhou, C.; Zhang, S.; Lv, Z.; Ma, Z.; Yu, C.; Zhihong, F.; Chan, M. Self-driven WSe 2 photodetectors enabled with asymmetrical van der Waals contact interfaces. npj 2D Mater. Appl. 2020, 4, 1-9. [CrossRef]

66. Kumar, M.; Lim, J.; Kang, H.; Kim, S.; Seo, H. Photon-triggered self-powered all electronics with graphene-silicon hybrid device. Nano Energy 2021, 82, 105668. [CrossRef]

67. Zhang, T.; Yang, T.; Zhang, M.; Bowen, C.R.; Yang, Y. Recent Progress in Hybridized Nanogenerators for Energy Scavenging. iScience 2020, 23, 101689. [CrossRef]

68. Pan, C.; Zhai, J.; Wang, Z.L. Piezotronics and Piezo-phototronics of Third Generation Semiconductor Nanowires. Chem. Rev. 2019, 119, 9303-9359. [CrossRef]

69. Fan, F.; Wu, W. Emerging Devices Based on Two-Dimensional Monolayer Materials for Energy Harvesting. Research 2019, 2019, 7367828. [CrossRef] 
70. Kim, S.K.; Bhatia, R.; Kim, T.-H.; Seol, D.; Kim, J.H.; Kim, H.; Seung, W.; Kim, Y.; Lee, Y.H.; Kim, S.-W. Directional dependent piezoelectric effect in CVD grown monolayer MoS2 for flexible piezoelectric nanogenerators. Nano Energy 2016, 22, 483-489. [CrossRef]

71. Kuang, H.; Li, Y.; Huang, S.; Shi, L.; Zhou, Z.; Gao, C.; Zeng, X.; Pandey, R.; Wang, X.; Dong, S.; et al. Piezoelectric boron nitride nanosheets for high performance energy harvesting devices. Nano Energy 2021, 80, 105561. [CrossRef]

72. Dai, M.; Zheng, W.; Zhang, X.; Wang, S.; Lin, J.; Li, K.; Hu, Y.; Sun, E.; Zhang, J.; Qiu, Y.; et al. Enhanced Piezoelectric Effect Derived from Grain Boundary in MoS2 Monolayers. Nano Lett. 2019, 20, 201-207. [CrossRef]

73. Wang, Z.L. Triboelectric nanogenerators as new energy technology and self-powered sensors-Principles, problems and perspectives. Faraday Discuss. 2014, 176, 447-458. [CrossRef]

74. Park, S.; Park, J.; Kim, Y.-G.; Bae, S.; Kim, T.-W.; Park, K.-I.; Hong, B.H.; Jeong, C.K.; Lee, S.-K. Laser-directed synthesis of strain-induced crumpled MoS2 structure for enhanced triboelectrification toward haptic sensors. Nano Energy 2020, 78, 105266. [CrossRef]

75. Seol, M.; Kim, S.; Cho, Y.; Byun, K.-E.; Kim, H.; Kim, J.; Kim, S.K.; Kim, S.-W.; Shin, H.-J.; Park, S. Triboelectric Series of 2D Layered Materials. Adv. Mater. 2018, 30, 1801210. [CrossRef]

76. Oh, J.Y.; Lee, J.H.; Han, S.W.; Chae, S.S.; Bae, E.J.; Kang, Y.H.; Choi, W.J.; Cho, S.Y.; Lee, J.O.; Baik, H.K.; et al. Chemically exfoliated transition metal dichalcogenide nanosheet-based wearable thermoelectric generators. Energy Environ. Sci. 2016, 9 , 1696-1705. [CrossRef]

77. Jeong, Y.; Shin, D.; Park, J.; Park, J.; Yi, Y.; Im, S. Integrated advantages from perovskite photovoltaic cell and 2D MoTe 2 transistor towards self-power energy harvesting and photosensing. Nano Energy 2019, 63, 103833. [CrossRef]

78. Sun, J.; Chang, Y.; Dong, L.; Zhang, K.; Hua, Q.; Zang, J.-H.; Chen, Q.; Shang, Y.; Pan, C.; Shan, C. MXene enhanced self-powered alternating current electroluminescence devices for patterned flexible displays. Nano Energy 2021, 86, 106077. [CrossRef]

79. Roy, K.; Jana, S.; Mallick, Z.; Ghosh, S.K.; Dutta, B.; Sarkar, S.; Sinha, C.; Mandal, D. Two-Dimensional MOF Modulated Fiber Nanogenerator for Effective Acoustoelectric Conversion and Human Motion Detection. Langmuir ACS J. Surf. Colloids 2021, 37, 7107-7117. [CrossRef]

80. Lee, Y.; Kim, J.; Jang, B.; Kim, S.H.; Sharma, B.K.; Kim, J.-H.; Ahn, J.H. Graphene-based stretchable/wearable self-powered touch sensor. Nano Energy 2019, 62, 259-267. [CrossRef]

81. Shi, K.; Jinhua, L.; Xiao, Y.; Liang, G.; Chu, X.; Zhai, Y.; Zhang, B.; Lu, D.; Rosei, F. High-Response, Ultrafast-Speed, and Self-Powered Photodetection Achieved in InP@ZnS-MoS 2 Phototransistors with Interdigitated Pt Electrodes. ACS Appl. Mater. Interfaces 2020, 12, 31382-31391. [CrossRef]

82. Zhou, G.; Ren, Z.; Wang, L.; Wu, J.; Sun, B.; Zhou, A.; Zhang, G.; Zheng, S.; Duan, S.; Song, Q. Resistive switching memory integrated with amorphous carbon-based nanogenerators for self-powered device. Nano Energy 2019, 63, 103793. [CrossRef] 